\title{
DETERMINING FEASIBILITY OF A PROPULSIONLESS MICROSATELLITE FORMATION FLIGHT MISSION
}

\author{
A Thesis \\ presented to \\ the Faculty of California Polytechnic State University, \\ San Luis Obispo
}

\author{
In Partial Fulfillment \\ of the Requirements for the Degree \\ Master of Science in Aerospace Engineering
}

by

Aaron A. Levis

June 2018 
(C) 2018

Aaron A. Levis

ALL RIGHTS RESERVED 


\section{COMMITTEE MEMBERSHIP}

TITLE:

Determining Feasibility of a Propulsionless

Microsatellite Formation Flight Mission

AUTHOR:

Aaron A. Levis

DATE SUBMITTED: June 2018

COMMITTEE CHAIR: Amelia Greig, Ph.D.

Cal Poly Aerospace Engineering Dept.

COMMITTEE MEMBER: Roderick Boswell, Ph.D.

ANU Space Plasma Power and Propulsion Group.

COMMITTEE MEMBER: Eric Mehiel, Ph.D.

Cal Poly Aerospace Engineering Dept.

COMMITTEE MEMBER: Joseph Carpico

Cal Poly Aerospace Engineering Dept. 


\begin{abstract}
Determining Feasibility of a Propulsionless Microsatellite Formation Flight Mission
\end{abstract}

Aaron A. Levis

Benefits of developing missions with multiple formation flying spacecraft as an alternative to a traditional monolithic vehicle are becoming apparent. In some cases, these missions can lower cost and increase flexibility among other situational advantages. However, there are various limitations that are imposed by these missions that are centered on the concept of maintaining the necessary formation. One such limitation is that of the propulsion system required for each spacecraft. To mitigate the complexity and mass of the onboard propulsion, the pairing of electromagnetic actuators and differential drag to replace the functionality of a propulsive system is investigated. By using COTS magnetorquer boards to command satellite orientation, a scenario in which two 3U CubeSats are initially deployed from the ISS NanoRacks at an altitude of $400 \mathrm{~km}$. They are then commanded to achieve a relative separation of $1 \mathrm{~km}$ and hold the spacing to demonstrate the capability of formation flight. The scenario was simulated through the MATLAB/Simulink platform and the magnitude of the necessary command torques were determined. By comparison to the ISIS magnetorquer board, the necessary command torques seem relatively high than compared to what the actuator is capable of. The ISIS board may supply $\sim 5 \mathrm{e}-6 \mathrm{Nm}$ of torque while the mission requires as much as 3e-3 Nm at times. However, by extending the settling time of the control law at the expense of absolute orientation control, the control torques necessary to carry out the simulated mission are well within the bounds of the ISIS magnetorquer boards as well as other COTS boards. With this alteration, mission feasibility is determined. It should be noted that further analysis should be conducted regarding concerns with CubeSat detumble to further confirm feasibility. 


\section{ACKNOWLEDGMENTS}

Thanks to:

- Dr. Amelia Greig for providing me with the support I needed to march forth. With your guidance, my progress was mediated and expedited in order to produce work that I am proud to have completed. From my initial undertaking of the project to the last simulation run, my transformation as a student and as a professional are most forwardly thanks to you.

- Dr. Roderick Boswell for stimulating the inception and advancement of this project. Your inquiries provided the direction necessary for me to stay focused and determined. I feel privileged to have been able to work with you over the last year dissecting a problem that we share a common interest in. Your mentorship has been unique to my academic career and for this I give thanks.

- Dr. Eric Mehiel for providing me with a fulfilling academic career. The courses you have have led and lectured have been the cornerstone of my experiences at Cal Poly. It is from these lessons that I will carry a fortified toolkit into my professional career. The knowledge you have provided over the entirety of my education is invaluable and I look forward to continuing in the field of controls engineering. Thank you for being a superb role model.

- Joe for passing on your extensive industry knowledge. I am so grateful to have been able to learn from you over the last two years. From your lessons I feel more apt to enter a professional role and succeed outright. Your unique experiences were a valuable addition to my own academic composition as well as the Cal Poly aerospace faculty.

- The entirety of the Cal Poly aerospace faculty for fostering a rigorous and exciting program. 


\section{TABLE OF CONTENTS}

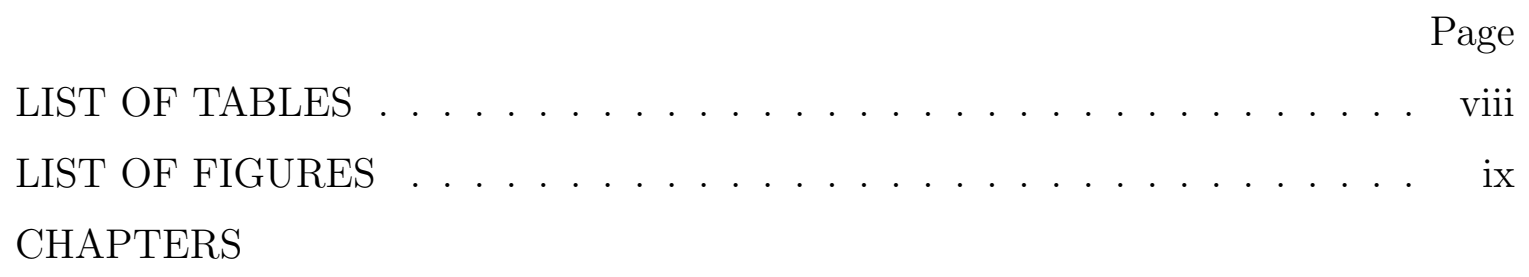

1 Introduction . . . . . . . . . . . . . . . . . 1

1.1 Previous Work .......................... 1

1.2 Magnetorquers ...................... 3

1.3 Differential Drag . . . . . . . . . . . . . . 5

1.4 Formation Flight . . . . . . . . . . . . . . . 6

1.5 Perturbations ....................... 7

1.6 Coordinate Transformations . . . . . . . . . . . . . . . 11

2 Simulation . . . . . . . . . . . . . . . . . . 13

2.1 Matlab/Simulink Implementation . . . . . . . . . . . . . 13

2.2 Space Environment Model . . . . . . . . . . . . . . . . . . 14

2.2.1 Drag Model . . . . . . . . . . . . . 15

2.2.2 SRP Model . . . . . . . . . . . . . . 17

2.2.3 Earth Oblateness Model . . . . . . . . . . . . . . 18

2.2.4 3-Body Model . . . . . . . . . . . . . . . . . . . 19

2.3 Initial Conditions . . . . . . . . . . . . . . . 20

2.3.1 NanoRack Deployment . . . . . . . . . . . . 21

2.3.1.1 Relative Velocity ............. 21

2.3.1.2 Orbit Circularization . . . . . . . . . . 21

2.4 Model Constraints . . . . . . . . . . . . . . . . . 22

3 System Control . . . . . . . . . . . . . . . . . . 23

3.1 PD Control . . . . . . . . . . . . . . . . . . . . . . 23

3.2 Timing Control . . . . . . . . . . . . . . 25

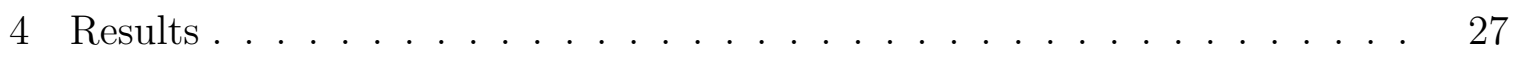

4.1 Control Verification . . . . . . . . . . . . . . . 27

4.2 Spacecraft Altitude . . . . . . . . . . . . . . . 29 
4.3 Relative Position . . . . . . . . . . . . . . . . . . . . . 30

4.4 Control Torques . . . . . . . . . . . . . . . . . . . . . . . . . . 31

4.5 Alternative Solutions . . . . . . . . . . . . . . . 36

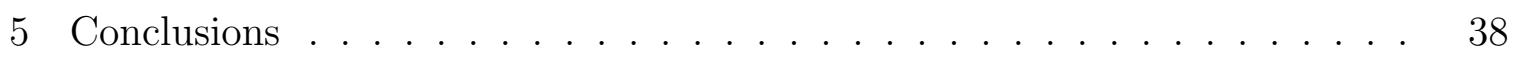

5.1 Discussion . . . . . . . . . . . . . . . . 38

5.1.1 Spacecraft Characteristics . . . . . . . . . . . 38

5.1 .2 Control Torques . . . . . . . . . . . . . . . . . . . . . 39

5.1 .3 Detumble . . . . . . . . . . . . . . . . . . . 39

5.1 .4 Perturbations . . . . . . . . . . . . . . . . 40

5.2 Future Work . . . . . . . . . . . . . . . . . . . . . . . 41

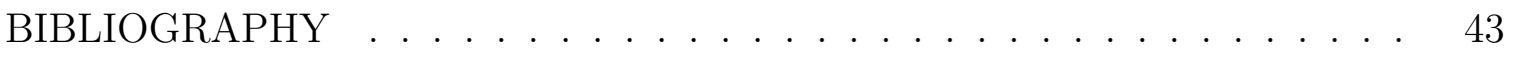
APPENDICES

A Complete Results From Primary Simulation . . . . . . . . . . 47

B Complete Results From Alternative Simulation . . . . . . . . . . . . 51 


\section{LIST OF TABLES}

Table

Page

2.1 Atmospheric density for the first two days of mission. . . . . . . 15

2.2 Initial satellite orbital elements. . . . . . . . . . . . . . . 20

3.1 Spacecraft control parameters. . . . . . . . . . . . 25

3.2 Mission schedule. . . . . . . . . . . . . . 26

4.1 Quaternion Commands . . . . . . . . . . . . . 27

4.2 Alternative spacecraft control parameters. . . . . . . . . . . 33

4.3 Alternative mission schedule. . . . . . . . . . . . . . . 33

5.1 Sapcecraft Characteristics . . . . . . . . . . . . . . . 39

5.2 Sapcecraft Characteristics . . . . . . . . . . . . . 39 


\section{LIST OF FIGURES}

Figure

Page

$1.13 \mathrm{U}$ CubeSat ISIS Magnetorquer Board.[1] . . . . . . . . . 4

1.2 3U CubeSat Geometry . . . . . . . . . . . . . . . 5

1.3 3U CubeSat minimum drag configuration. . . . . . . . . . . . 6

$1.43 \mathrm{U}$ CubeSat maximum drag configuration. . . . . . . . . . . 6

1.5 ECI, LVLH, and Body Frame coordinate systems. . . . . . . . . . 11

2.1 Complete Simulink model of satellite dynamics and control. . . . . 14

2.2 Accelerations due to drag over first two days of mission. . . . . . . 16

2.3 Accelerations due to SRP over first two days of mission. . . . . . . 17

2.4 Accelerations due to Earth's oblateness over first two days of mission. 18

2.5 Spacecraft accelerations due to 3-body system over first two days of mission. . . . . . . . . . . . . . . . . . . 19

2.6 Finite differencing for adaptive Runge Kutta 45 method. . . . . . . 22

4.1 Quaternion components over mission lifetime. . . . . . . . . 28

$4.2 \quad$ Altitude of spacecraft A over mission lifetime. . . . . . . . . . . . . 29

4.3 Relative position of spacecraft over mission lifetime. . . . . . . . . . 30

4.4 Spacecraft control torques over mission lifetime. . . . . . . . . . . . 31

4.5 Spacecraft control torques during nominal phase. . . . . . . . . . . 32

4.6 Relative position of spacecraft for alternative mission. . . . . . . . . 34

4.7 Control torque magnitudes for alternative mission. . . . . . . . . . 34

4.8 Quaternion components for alternative mission. . . . . . . . . . 35

4.9 MAI 400 Miniature Attitude Determination and Control System.[2] 37

5.1 Periodicity in relative position. . . . . . . . . . . . . 41

A.1 Relative position of spacecraft over mission lifetime. . . . . . . . . 47

A.2 Accelerations due to drag over mission lifetime. . . . . . . . . . . . 48

A.3 Accelerations due to SRP over mission lifetime. . . . . . . . . . . 48

A.4 Accelerations due to Earth oblateness over mission lifetime. . . . . 49 
A.5 Accelerations due to 3-body system over mission lifetime. . . . . . . 49

A.6 Spacecraft quaternions over mission lifetime. . . . . . . . . . . . 50

A.7 Control torques required over mission lifetime. . . . . . . . . . . 50

B.1 Relative position of spacecraft over mission lifetime. . . . . . . . . 51

B.2 Accelerations due to drag over mission lifetime. . . . . . . . . . 52

B.3 Accelerations due to SRP over mission lifetime. . . . . . . . . . . 52

B.4 Accelerations due to Earth oblateness over mission lifetime. . . . . 53

B.5 Accelerations due to 3-body system over mission lifetime. . . . . . 53

B.6 Spacecraft quaternions over mission lifetime. . . . . . . . . . 54

B.7 Control torques required over mission lifetime. . . . . . . . . . 54 


\section{Chapter 1}

\section{INTRODUCTION}

The main focus of this study is to model with medium to high fidelity the complex satellite dynamics incorporating magnetorquer ADCS. The electromagnetic fields produce will allow for varying spacecraft geometries to induce differential drag for the maintenance of formation flight.

\subsection{Previous Work}

Within the last decade there have been several missions which utilize multiple smaller spacecraft as an alternative to a traditional monolithic spacecraft. Some of the benefits of this approach include but are not limited to: synthesis of large sensor apertures, mission flexibility, increased reliability, upgradeability, staged deployment, and lower costs[3]. This ideology is exemplified through the missions of CLUSTER and tanDEM-X. In order to achieve the science objectives for the CLUSTER mission, the European Space Agency (ESA) placed four identical spacecraft in a tetrahedral configuration to better measure the 3-dimensional structure of the magnetosphere[4]. Similarly, tanDEM-X involves two satellites at close proximity flying in a helical formation[5].

While distributed satellite formations offer several advantages over large multiinstrumented spacecraft for earth and space science applications, there are a number of significant technical challenges that must be overcome to transition these systems from concept to reality. Current approaches to meet these challenges include the use of global positioning systems for satellite tracking and navigation, au-

tonomous spacecraft control algorithms to correlate observations and maintain precise 
inter-satellite separations, and electric or chemical microthrusters to provide satellite station-keeping, drag make-up, and array reconfiguration[6]. Each of these techniques, while promising, have inherent limitations that make their implementation exceedingly difficult to put into practice. Electric propulsion systems, such as pulsed plasma thrusters, can provide the fine control required for drag makeup and station-keeping but will compete for limited spacecraft power. Chemical microthrusters can provide higher thrust than their electric thruster counterparts, but use more propellant due to their lower specific impulse values, resulting in a larger propellant mass fraction or a reduced operational life. In addition, potential contamination of neighboring spacecraft by propellant exhaust plumes and the possibility of pulsed electromagnetic interference with low power inter-satellite communications remain a real concern for tightly grouped clusters[6].

To mitigate the complexity of a propulsion system onboard a formation flying spacecraft, the concept of electromagnetic formation flight (EMFF) was developed. EMFF is a recent concept, aiming to control relative motions of formation flying satellites using magnetic interactions[7]. By equipping satellites with an electromagnetic actuator, each spacecraft can then create a dipole that interacts with the Earth's magnetic field and other spacecraft dipoles, inducing a desired torque that can be used to control the relative positions of the vehicles within the formation.

The study put forth in this paper has heritage from several of these concepts being discussed. The scenario being simulated stems from some of the EMFF applications being researched in current missions. Each spacecraft being modeled is void of a propulsive system and relies entirely on magnetorquers for GN\&C. By omitting onboard propulsion, the CubeSats being modeled have reduced mass and complexity. In previous missions, the use of electromagnetic actuation is used solely for the purpose of maintaining the necessary orientation of each spacecraft within a constellation. However, the study being presented pairs EMFF with aerodynamic forces to execute 
orbital maneuvers. The study will determine if the use of differential drag forces will appropriate the mitigation of a propulsive device while still enabling the option of formation flight spatial alteration. Agreeably, studies focusing on CubeSat formation flight through differential drag have conceptual traction through extensive knowledge of CubeSat drag and deorbit analysis[8]. This paper aims at validating the feasibility of commanding the orientations necessary for achieving orbital separation by way of COTS electromagnetic actuators.

\subsection{Magnetorquers}

One concept imperative to this project is that of electromagnetic actuator implementation. Electromagnetic actuators have been used on spacecraft for the past 50 years[9]. Magnetorquers, or torque rods, flow current through an electromagnetic coil to produce a dipole field. This dipole will then interact with the Earth's magnetic field to induce a torque on a spacecraft. These actuators has been used extensively in spacecraft ADCS applications including the desaturation of reaction wheels in a process known as momentum unloading[10].

In the case being studied, the 3U CubeSats being modeled will alter their orientations by the use of these magnetorquers. In order to verify the feasibility of the system, the magnitude of the control torques necessary to commit these maneuvers must be less than or equal to that which can be provided by COTS magnetorquer boards. Such boards measure performance in $A \mathrm{~m}^{2}$ designating the magnitude of the dipole moment supplied. The torque that can then be provided by these boards is determined by the specific orbit at which the device is operated. This relationship is shown below in Equation 1.1[11] where $\tau$ is the torque that is provided in $N m$ and $B$ is the Earth's magnetic field measured in Teslas.

$$
\tau=A m^{2} \cdot B
$$


To determine the amount of torque available from the magnetorquer, first one board must be selected. In Fig. 1.1, a commonly used ISIS CubeSat magnetorquer board is pictured. This magnetorquer board can be used on 3U CubeSat missions and supplies the spacecraft with a maximum dipole moment of $0.2 \mathrm{Am}^{2}$. For COTS magnetorquer boards $0.2 \mathrm{Am}^{2}$ is accepted to be the upper limit of performance. This allows the ISIS board to be a valid representation of what could used when transitioning the simulated mission to a space application.

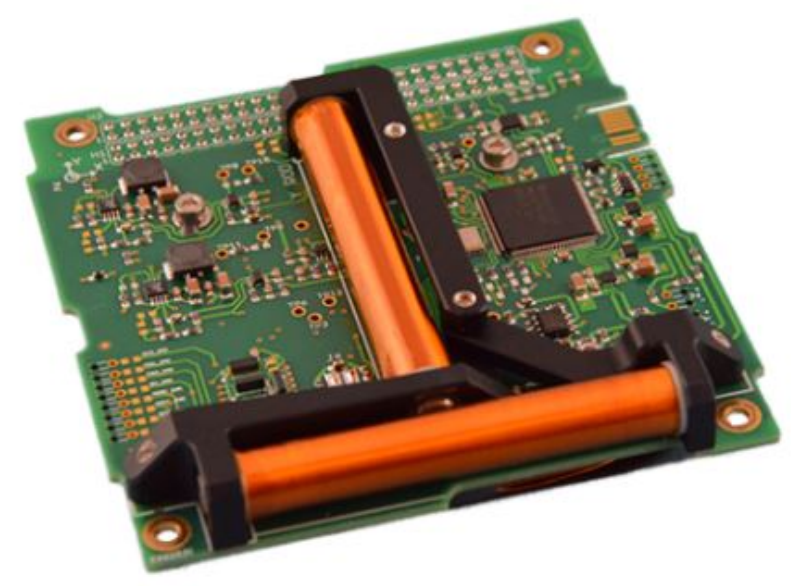

Figure 1.1: 3U CubeSat ISIS Magnetorquer Board.[1]

While the magnitude of Earth's magnetic field varies throughout altitude as well as time, the mission being modeled is carried out is at an altitude of $400 \mathrm{~km}$ where the magnetic field is roughly $26,000 n T[12]$. When combining the magnetic field at this altitude with the dipole moment available from the magnetorquers, it can then be determined that the $3 \mathrm{U}$ CubeSat will be able to execute maneuvers with a maximum torque magnitude of $5.2 \mathrm{e}-6 \mathrm{Nm}$. In order to verify the feasibility of the mission, the control torques necessary to command the orientations necessary to induce differential drag as well as overcome orbital perturbations must not exceed the magnitude of available torque. 


\subsection{Differential Drag}

Differential drag makes use of differing surface areas between spacecraft, causing varying drag force to be experienced, and thus changing the spacecraft motion. The effect of surface area on the drag force experienced by a spacecraft is made evident in Equation 1.8[13] where $A$ is the surface area normal to the velocity vector of the spacecraft.

$$
F_{D}=\frac{1}{2} C_{D} A \rho V^{2}
$$

This drag force is then modeled as one factor in the spacecraft motion shown by Equation 1.3.

$$
\ddot{r}_{d}=\frac{1}{2 m} C_{D} A \rho V^{2}
$$

By controlling the orientations of the spacecraft that expose alternating surface areas in the v-bar direction, desired motion can be commanded. In the case of this study, two $3 \mathrm{U}$ CubeSats are being modeled in the simulation with a simplified geometry shown in Fig. 1.2 with units in meters and the reference origin being that of the spacecraft body frame.

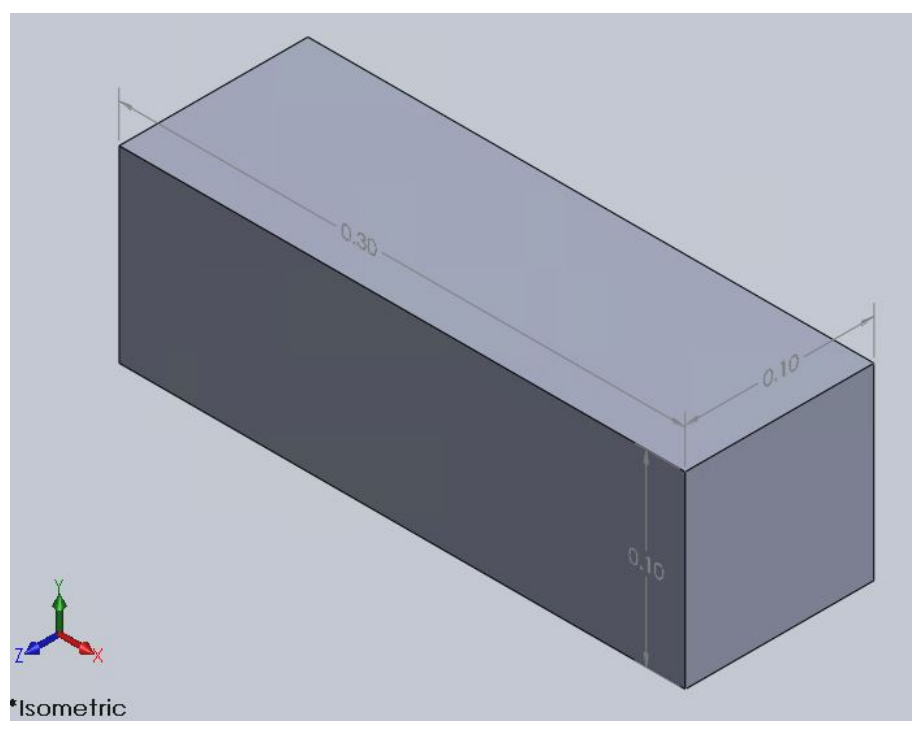

Figure 1.2: 3U CubeSat Geometry 
By commanding various CubeSat orientations, the surface areas exposed to be normal to the velocity vector are shown in the figures below. To minimize the drag force on the satellite, the vehicle is controlled to maintain the orientation in Fig. 1.3 with an incident surface area of $.01 \mathrm{~m}^{2}$. To maximize the drag force on the satellite and lower its orbital altitude, the satellite is controlled to maintain the orientation in Fig. 1.4 with an incident surface area of $.03 \mathrm{~m}^{2}$.

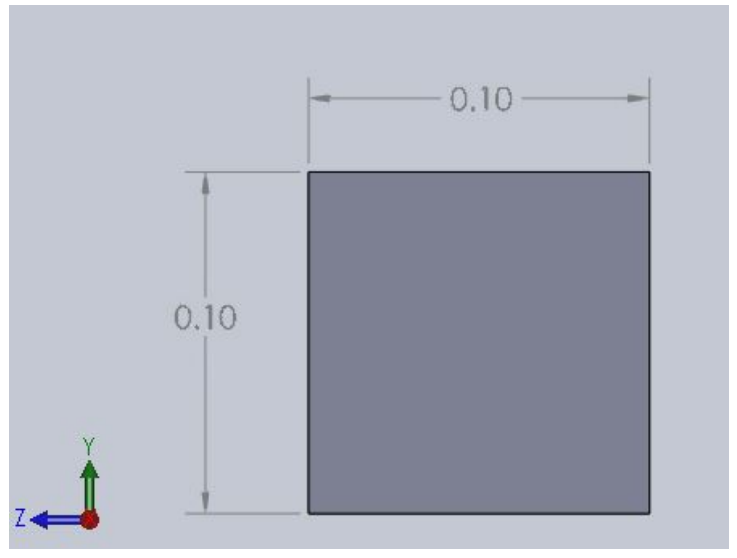

Figure 1.3: 3U CubeSat minimum drag configuration.

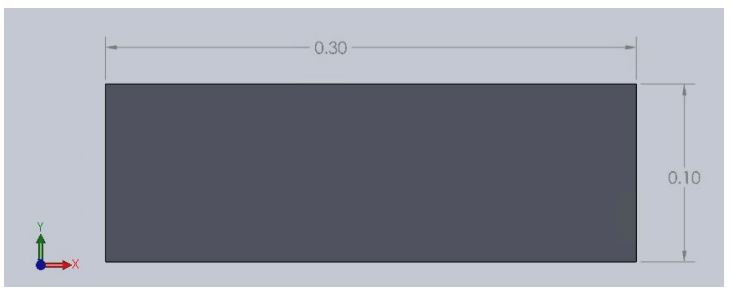

Figure 1.4: 3U CubeSat maximum drag configuration.

By altering their orientations, the two spacecraft aim to achieve a relative separation of $1 \mathrm{~km}$ and then hold this distance. If the two spacecraft are able to execute these maneuvers through use of magnetorquers, the feasibility of a propulsionless microsatellite formation flight mission may be confirmed.

\subsection{Formation Flight}

The concept of formation flight has come to have a wide range of definitions throughout its development in various missions. Most loosely, formation flight must consist of one or more chaser satellites that utilize active control in order to maintain a desired relative position to a passive target satellite[7]. The nonlinear equations of motion for both spacecraft are functions of their spatial location and the forces acting on them 
at that point[13]. While the target spacecraft motion is uncontrolled and governed by these forces, the chaser spacecraft utilizes a propulsion system to apply an external force and alter its relative distance to a target location. This propulsive force is represented by $\mathbf{u}$ in Equation 1.5[14] where the subscript 2 designates the chaser satellite. However, the omission of this propulsion is shown in Equation 1.4[14] where the subscript 1 designates the target satellite. It should also be noted here that only magnitude of a variable is to be assumed if no vector notation is made clear in the indicated equation.

$$
\begin{gathered}
\ddot{\vec{r}}_{1}=f\left(\vec{r}_{1}\right) \\
\ddot{\overrightarrow{r_{2}}}=f\left(\vec{r}_{2}\right)+\vec{u}
\end{gathered}
$$

The propulsive force $\vec{u}$ will force $\delta \vec{r}$, shown in Equation 1.6[14], to be driven to zero as the position of the chaser satellite, $\vec{r}_{2}$, is driven to the desired position, $\vec{r}_{2_{d}}$.

$$
\delta \vec{r}=\vec{r}_{2}-\vec{r}_{2_{d}}
$$

However, the purpose of this study is to eliminate the propulsive force $\mathbf{u}$ and use the orientation of the spacecraft to alter the dynamics of the nonlinear motion that the spacecraft experiences. This simplifies Equation 1.5 to omit the use of propulsion as shown in Equation 1.7.

$$
\ddot{\overrightarrow{r_{2}}}=f\left(\vec{r}_{2}\right)
$$

In order to model the complex dynamics contained within Equation 1.7, several orbital perturbations must be included.

\subsection{Perturbations}

In order to model the mission with medium to high fidelity, the inclusion of orbital perturbations and the accuracy at which they are calculated is vital. In the model, 
drag, solar radiation pressure (SRP), and Earth oblateness are modeled to alter the spacecraft motion while propagating the motion in a 3-body system to include the effects of the sun on spacecraft acceleration.

For the purposes of this study, the most important perturbation being modeled is that of drag for several reasons. Firstly, due to the fact that the mission being modeled in Low Earth Orbit (LEO), aerodynamic drag will impose the largest magnitude perturbation on the spacecraft motion. Next, the success of the mission is reliant on accurately determining the magnitude of drag imposed on the $3 \mathrm{U}$ CubeSats being modeled. As such, the drag calculated by Equation 1.8[13] must be done so with high accuracy. To determine the drag perturbation, several parameters about the spacecraft must be known including $C_{D}$ the coefficient of drag, $A$ the area incident to the v-bar direction, $\rho$ being atmospheric density, and $V$ being the velocity of the spacecraft relative to Earth's rotation. For the purposes of this study a value of 4 was assigned for $C_{D}$ as it models a flat plate normal to the v-bar direction[15].

$$
\ddot{\vec{r}}_{d}=-\frac{1}{2 m} C_{D} A \rho V \cdot \vec{V}
$$

In addition to drag, SRP is being modeled. SRP is created by the highly energetic particles emitted by the Sun that travel towards Earth and collide with the spacecraft. The impulse of these particles then cause a small deceleration in the direction parallel and opposite to the direction of travel. In addition, the SRP perturbation is only apparent when the spacecraft is in daylight. Once the vehicle has entered eclipse, the Earth blocks the direction at which the particles are travelling and the value of the shadowing function $\nu$ becomes 0 . The acceleration that SRP creates when the spacecraft has line of sight to the sun and $\nu$ is equal to 1 is shown below in Equation 1.9[13]. The momentum flux of the solar radiation is designated by $P_{S R}$ and has a value of $4.56 e-6 \frac{N}{m^{2}}[13]$. The radiation pressure coefficient being $C_{R}$ has a value between 1 and 2 and is assigned a value of 1.2 for this specific simulation but 
is ultimately spacecraft specific. $A$ is the area exposed to the Sun, and $m_{S C}$ is the mass of the spacecraft. it should be noted that an assumption being made is that the acceleration due to SRP will be an average for the entirety of its pass through sunlight. This detracts from the fidelity of the simulation, but does not violate the validity of the model as SRP is not a dominant perturbation in LEO orbits.

$$
\ddot{\vec{r}}_{S R P}=-\nu \frac{P_{S R} C_{R} A}{m_{S C} r_{\oplus-\odot}} \vec{r}_{\oplus-\odot}
$$

Along with aerodynamic drag and SRP, Earth oblateness has a significant effect of spacecraft motion. Due to Earth's oblateness, there are zonal harmonics at which an orbiting spacecraft will deviate from its 2-body orbital path. These harmonics can be modeled with accuracy up to over 100 distinct zones at which the Earth's shape causes slightly different gravitational pull as the spacecraft passes[13]. These zones are represented by the letter $J$ followed the number of that divisional zone. For the purposes of this model, $J 2$ is the most concerning perturbation in need of modeling. In addition, the $J 3$ perturbation is modeled to increase fidelity. The equations for modeling these zones comes partially from experimentation, allowing the coefficients $J_{2}$ and $J_{3}$ to be constant where they are found in Equations 1.10[13] and 1.11[13]. These constant coefficients were found scientifically and are equivalent to $1.08262668355 e-3$ and $2.53265648533 e-6$ respectively[13]. The components of the calculated acceleration due to $J 2$ are shown here.

$$
\begin{aligned}
& \ddot{r}_{J 2 x}=-\frac{3}{2} \frac{J_{2} \mu r_{\oplus}^{2} r_{x}}{r^{5}}\left(1-\frac{5 r_{z}^{2}}{r^{2}}\right) \\
& \ddot{r}_{J 2 y}=-\frac{3}{2} \frac{J_{2} \mu r_{\oplus}^{2} r_{y}}{r^{5}}\left(1-\frac{5 r_{z}^{2}}{r^{2}}\right) \\
& \ddot{r}_{J 2 z}=-\frac{3}{2} \frac{J_{2} \mu r_{\oplus}^{2} r_{z}}{r^{5}}\left(3-\frac{5 r_{z}^{2}}{r^{2}}\right)
\end{aligned}
$$


With the $J 3$ zonal perturbations calculated slightly differently as displayed here.

$$
\begin{array}{r}
\ddot{r}_{J 3 x}=-\frac{5}{2} \frac{J_{3} \mu r_{\oplus}^{3} r_{x}}{r^{7}}\left(3 r_{z}^{2}-\frac{7 r_{z}^{3}}{r^{2}}\right) \\
\ddot{r}_{J 3 y}=-\frac{5}{2} \frac{J_{3} \mu r_{\oplus}^{3} r_{y}}{r^{7}}\left(3 r_{z}^{2}-\frac{7 r_{z}^{3}}{r^{2}}\right) \\
\ddot{r}_{J 3 z}=-\frac{5}{2} \frac{J_{3} \mu r_{\oplus}^{3}}{r^{7}}\left(6 r_{z}^{2}-\frac{7 r_{z}^{4}}{r^{2}}-\frac{3}{5} r^{2}\right)
\end{array}
$$

Combined together the acceleration due to $J 2$ is determined in Equation 1.12.

$$
\ddot{\vec{r}}_{J 2}=\left[\begin{array}{c}
\ddot{r}_{J 2 x} \\
\ddot{r}_{J 2 y} \\
\ddot{r}_{J 2 z}
\end{array}\right]
$$

With the acceleration due to $J 3$ shown here in Equation 1.13.

$$
\ddot{\vec{r}}_{J 3}=\left[\begin{array}{c}
\ddot{r}_{J 3 x} \\
\ddot{r}_{J 3 y} \\
\ddot{r}_{J 3 z}
\end{array}\right]
$$

Finally, it is necessary to model the spacecraft motion within the 3-body system it inhabits. This system involving the spacecraft, the Earth, and the Sun is modeled below in Equation 1.14[13] where $G$ is the gravitational constant, $m_{\odot}$ is the mass of the Sun, Earth is represented by the symbol $\oplus$, and $\vec{r}_{1-2}$ designates the vector from object 1 towards object 2 with SC being the spacecraft.

$$
\ddot{\overrightarrow{\vec{r}}}{ }_{3-b o d y}=G m_{\odot}\left(\frac{\vec{r}_{S C-\odot}}{r_{S C-\odot}^{3}}-\frac{\vec{r}_{\oplus-\odot}}{r_{\oplus-\odot}^{3}}\right)
$$

With these four orbital perturbations being modeled, the complete nonlinear system can be modeled as a sum of the accelerations. This transforms Equations 1.4 and 1.7 into Equations 1.15 and 1.16 as shown.

$$
\ddot{\vec{r}}_{1}=f\left(\vec{r}_{1}\right)=-\frac{\mu}{r_{1}^{3}} \vec{r}_{1}+\ddot{\vec{r}}_{3-b o d y_{1}}+\ddot{\vec{r}}_{d_{1}}+\ddot{\vec{r}}_{S R P_{1}}+\ddot{\vec{r}}_{J 2_{1}}+\ddot{\vec{r}}_{J 3_{1}}
$$




$$
\ddot{\vec{r}}_{2}=f\left(\vec{r}_{2}\right)=-\frac{\mu}{r_{2}^{3}} \vec{r}_{2}+\ddot{\vec{r}}_{3-b o d y_{2}}+\ddot{\vec{r}}_{d_{2}}+\ddot{\vec{r}}_{S R P_{2}}+\ddot{\vec{r}}_{J 2_{2}}+\ddot{\vec{r}}_{J 3_{2}}
$$

For this study, the difference in motion between the two spacecraft is determined within the $\ddot{\overrightarrow{\vec{r}_{d}}}$ term where the term $A$ is varying as the orientation of both spacecraft 1 and 2 is commanded to achieve the desired relative distance.

\subsection{Coordinate Transformations}

For the propagation of motion within the simulation, it is important to keep track of which reference frame in which the physics of the system is being accounted for. For this study, three reference frames are essential to determine the spacecraft dynamics. These three reference frames are the Earth Centered Interial frame (ECI), Local Vertical Local Horizon frame (LVLH), and the Body frame, all of which are defined and displayed in Fig. 1.5[16].

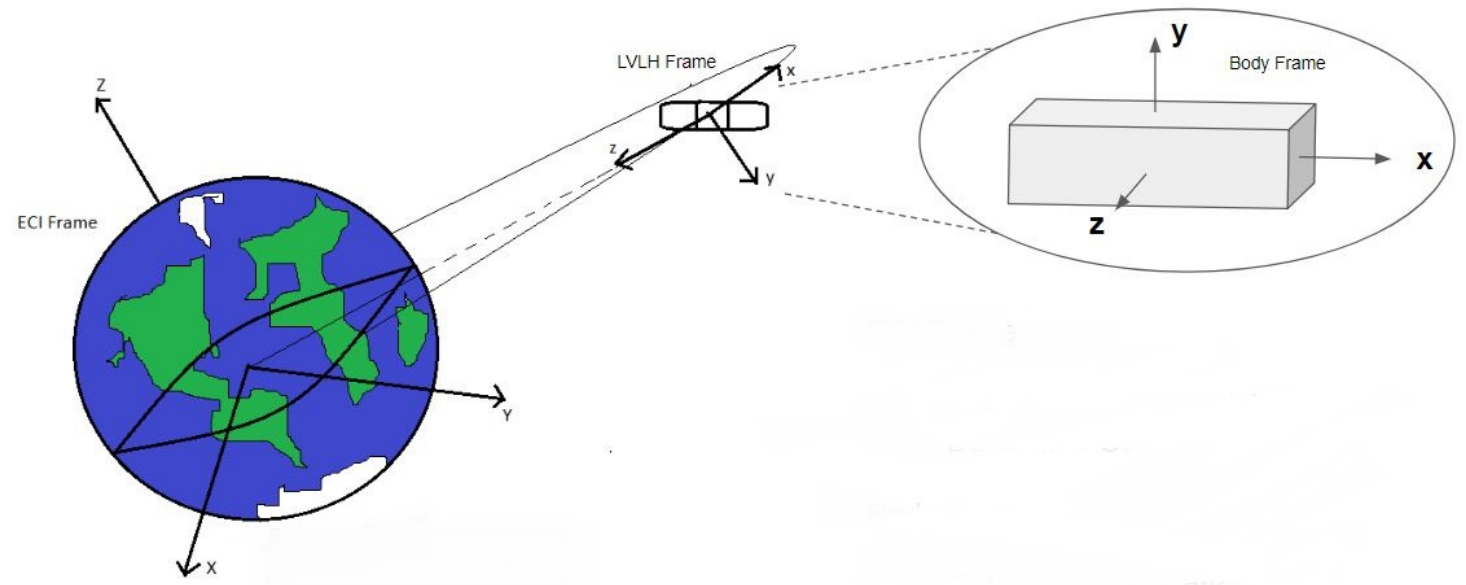

Figure 1.5: ECI, LVLH, and Body Frame coordinate systems.

While the ECI frame is necessary to determine orbital position along with the magnitude of the orbital perturbations at that point, the Body frame is necessary to determine the effects of disturbance torques on the spacecraft and the magnitude of the control torques necessary to apply. And finally, the LVLH frame allows the 
spacecraft to determine the orientation at which to fly to maintain the correct face in the v-bar direction. It is important to note the definition of both the LVLH and Body frames as they may vary when referencing alternative sources. Within the simulation modeled, the LVLH frame is defined to have the $\hat{z}$ unit vector pointing nadir, the $\hat{y}$ directed in the cross track direction, and the $\hat{x}$ unit vector completing the righthanded coordinate system. The Body frame is right-handed as well with the $\hat{x}$ unit vector pointing in the longitudinal axis of the $3 \mathrm{U}$ CubeSat and both the $\hat{y}$ and $\hat{z}$ unit vectors pointing in the latitudinal axes completing the system.

It also should be noted that all physics must be modeled within the same reference frame for the calculations to be correct. To propagate the orbital dynamics of the system, all vectors must be represented in the ECI frame. However, when looking at spacecraft torsional dynamics, all vectors must be represented within the Body frame. In addition, tracking the desired orientation of the spacecraft relative to its actual position requires the propagation of vectors in the LVLH frame. In order to keep track of the physics in each of these frames, it is necessary to switch between the representation of a vector in different frames. This is achieved through coordinate transformations[16].

The necessary coordinate transformations are made possible by the calculation of euler angles and, by extension, quaternions throughout the simulation. 
Chapter 2

SIMULATION

The mission being modeled is that of two $4 \mathrm{~kg} 3 \mathrm{U}$ CubeSats initially deployed with a relative position of $30 \mathrm{~cm}$ to each other. The mission goal is to use differential aerodynamic drag between the two spacecraft to acquire and maintain a relative position of $1 \mathrm{~km}$. In order to simulate the dynamics of the mission with medium to high fidelity, the space environment was modeled within the Simulink platform of Matlab.

\subsection{Matlab/Simulink Implementation}

With its massive collection of toolboxes and proven capability, Matlab was chosen as a means to build the model for the space environment and the simulated dynamics therein. Matlab works with the Simulink environment to provide a powerful tool for model-based design [17]. The Simulink platform allows for the user to build a model with whatever fidelity level is desired. For this study, a model with medium to high fidelity was achieved to determine feasibility with high confidence. The model constructed in Fig. 2.1 includes the satellite dynamics and control feedback for the mission.

As the figure suggests, the most basic segmentation of the model is into three main components: spacecraft dynamics, control determination, and system feedback. Within the blocks labeled Spacecraft $A$ and Spacecraft B lies the complete nonlinear model of the space environment and its effect on spacecraft linear and

torsional motion. From these blocks, the states of the spacecraft are fed into the Control Determination block by which the model determines the orientation at which 


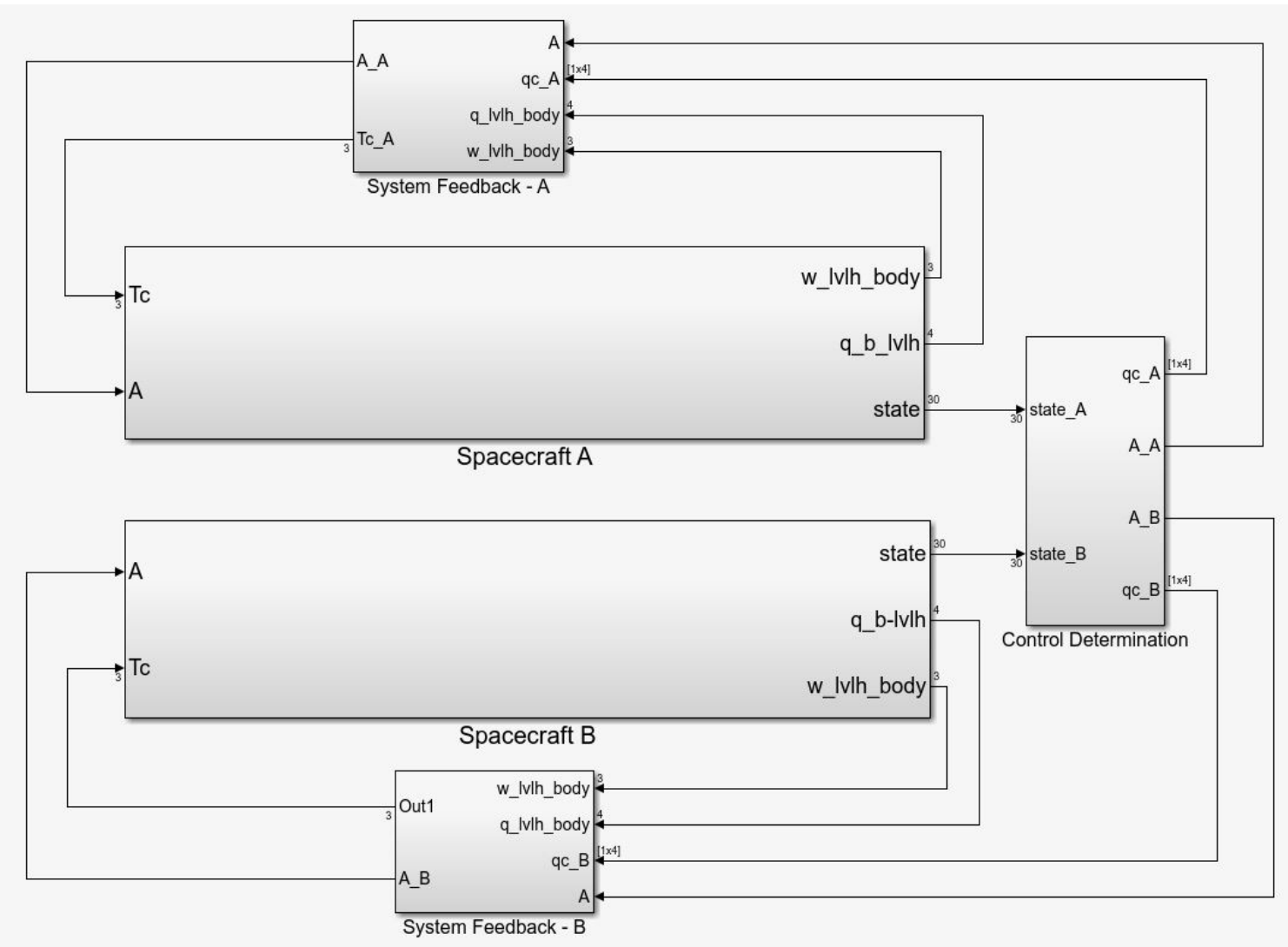

Figure 2.1: Complete Simulink model of satellite dynamics and control.

both spacecraft should be commanded. From this determination, commanded quaternions and resultant areas are fed back through both respective System Feedback blocks to determine the control torques necessary to maintain the desired orientations. It is through the magnitude of the control torques by which feasibility of the mission is determined.

\subsection{Space Environment Model}

The most essential goal of this study was to model the space environment with medium to high fidelity in order to provide accurate simulation results of spacecraft dynamics. To ensure the model created emulates the proper environment, several aspects of the model were analyzed and validated. To validate these aspects, 
the simulation was run for the first two days of the mission and the magnitudes of each perturbation experienced by the spacecraft were compared to the magnitudes of known models. It should be noted that the first maneuver in the simulated mission takes place at $\mathrm{T}+4$ hours after deployment at which time spacecraft A changes its orientation to the maximum drag configuration. This maneuver will be reflected in each of the perturbations tracked during this two day period.

\subsubsection{Drag Model}

The most essential variable in the calculation of the drag perturbation is the atmospheric density at the spacecraft altitude. While there are many atmospheric density models available for implementation, the 1976 exponential model for atmospheric density was chosen. The resultant density modeled over the first two days of the mission are displayed in Table 2.1[13].

Table 2.1: Atmospheric density for the first two days of mission.

\begin{tabular}{l|cc} 
& Satellite A & Satellite B \\
\hline Atmosheric Density $\left[\frac{\mathrm{kg}}{\mathrm{m}^{3}}\right]$ & $3.561 e-15$ & $3.561 e-15$ \\
\hline
\end{tabular}

Over the first two days, the satellites only alter their altitude by $30 \mathrm{~m}$ which does not constitute an alteration in the calculation of density within the 1976 exponential model. The magnitude of the density is consistent between both satellites due to their close proximity.

In addition to the calculation of density, a coefficient of drag must be chosen in order to calculate drag accelerations. For this mission, a coefficient of 4 was chosen to model a flat plat normal to the velocity vector. The resultant accelerations of both spacecraft due to drag are shown in Fig. 2.2. At 4 hours, the magnitude of the acceleration on Spacecraft A increases drastically indicating a successful change 


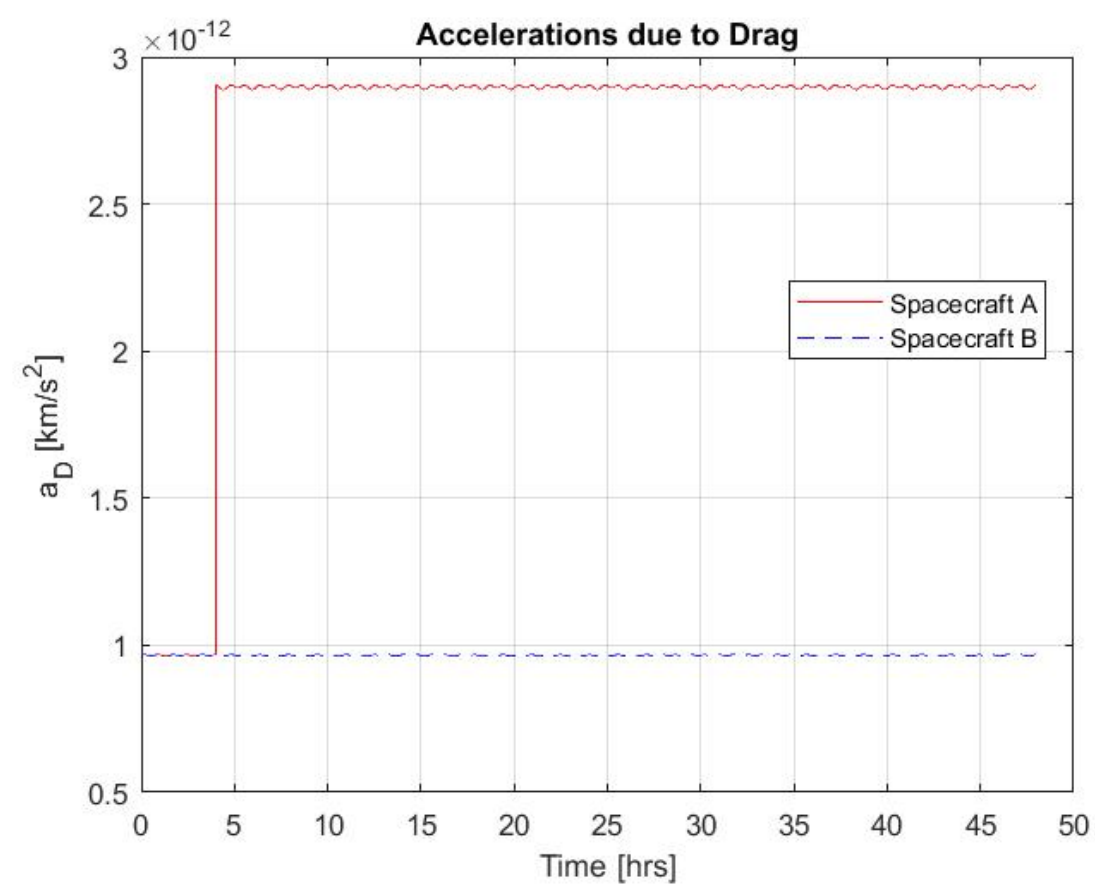

\section{Figure 2.2: Accelerations due to drag over first two days of mission.}

in spacecraft orientation to the maximum drag configuration as it is commanded at that time. The magnitudes of the accelerations are consistent with the density being determined by the 1976 exponential model as well as the drag formula described in Equation 1.3. As a result, the drag calculations can be validated within the model.

Over the course of time, the density at a given altitude is known to have both short term and long term fluctuations [15], those which are not captured by the model used. The argument is then made for the implementation of a higher fidelity density model. The main driver in the decision for utilizing the exponential model is that of computational time. While there are several density models including NRLMSISE-00 and JB2008 that provide more accurate calculations, the computational expense is too large for this application[15]. 


\subsubsection{SRP Model}

In order to determine accelerations due to SRP accurately, the position of the Sun relative to the Earth and spacecraft must be determined as well. This will cause the acceleration due to SRP to be zero when the spacecraft is in eclipse. The magnitudes of the SRP accelerations on both spacecraft are displayed in Fig. 2.3.
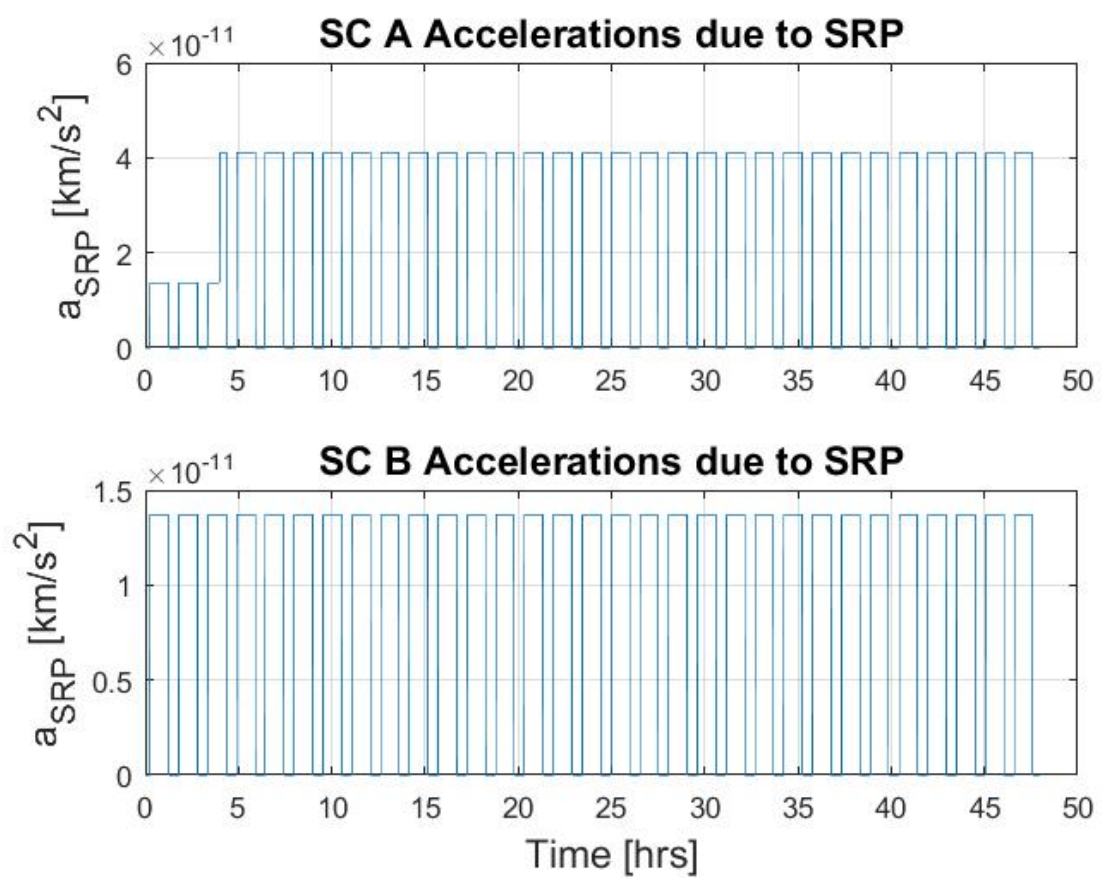

Figure 2.3: Accelerations due to SRP over first two days of mission.

Similar to the drag perturbation, the increase of the magnitude of SRP acceleration on spacecraft A increases after 4 hours. This increase is due to the orientation of spacecraft A commanding a larger surface area normal to the velocity in order to increase spacecraft separation. Also shown on the plot are discontinuities in acceleration effects due to the time of eclipse for both satellites. These times of eclipse last for roughly $1 / 3$ of each orbit in which the spacecraft are not affected by SRP. 


\subsubsection{Earth Oblateness Model}

Another orbital perturbation taken into account is that of Earth's oblateness. Because Earth is not perfectly spherical, small variations in the magnitude of gravity cause alterations in a spacecraft's orbit. The small accelerations due to this oblateness can be calculated using Equations 1.10 and 1.11. From implementing these equations within the simulation, the resulting accelerations on the CubeSats are shown in Fig. 2.4 .

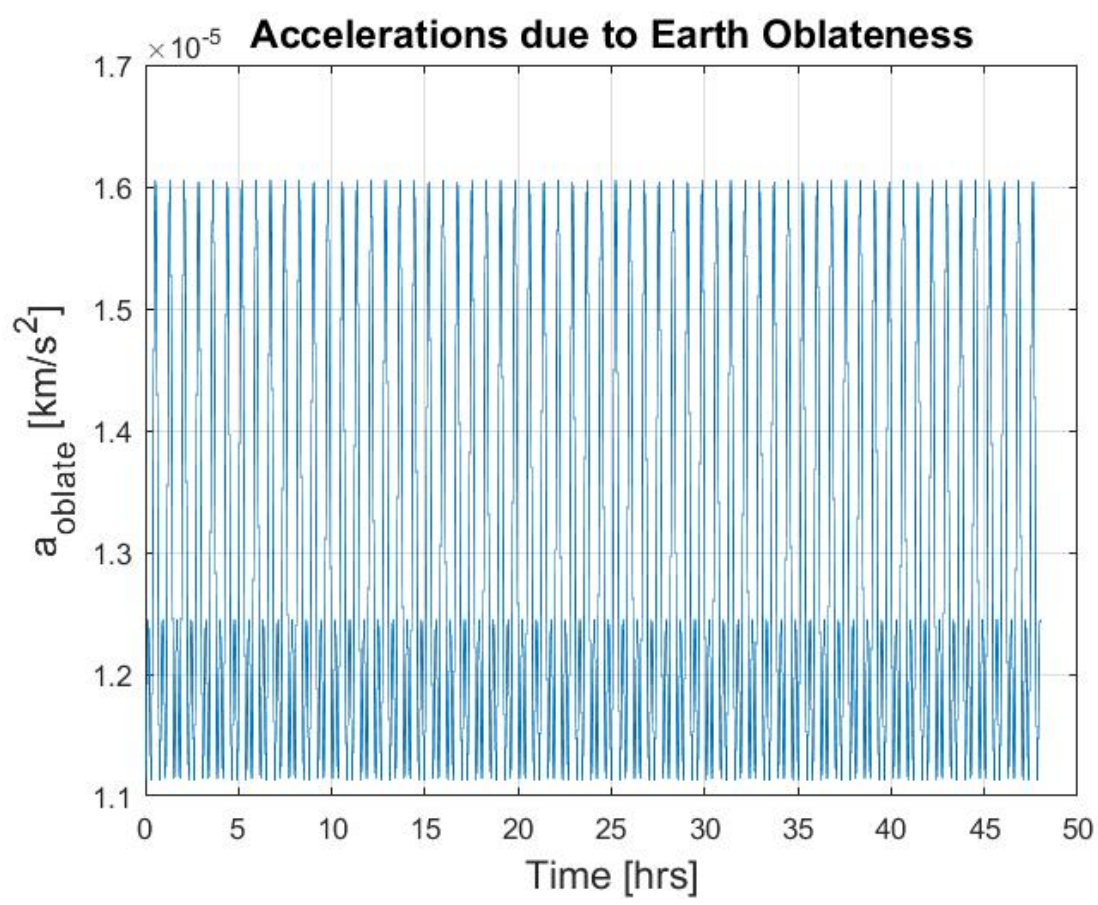

Figure 2.4: Accelerations due to Earth's oblateness over first two days of mission.

The periodicity of the perturbation is consistent with zonal modeling of the Earth's oblateness. As the spacecraft travel through different parts of their orbits, they experience different magnitudes of gravity at those points. While only spacecraft A is represented in the plot, the behavior of spacecraft $B$ is almost identical due to the close proximity of the vehicles. It is also important to note the relatively high magnitude of the accelerations as opposed to the SRP and drag perturbations. The 
inclusion of the $\mathrm{J} 2$ and $\mathrm{J} 3$ zonal harmonics are essential in simulating a high fidelity model for satellite dynamics.

\subsubsection{3-Body Model}

Another important perturbation to include is that of gravitational disturbances caused by the Sun. While low fidelity models will propagate spacecraft dynamics in a 2-body system involving only the satellite and the Earth, higher fidelity models will include other celestial bodies such as the Sun, Moon, or even Jupiter. To create a medium to high fidelity model, the 3-body system of the spacecraft, Earth, and Sun were modeled. The accelerations on the spacecraft within this system are shown in Fig. 2.5 .

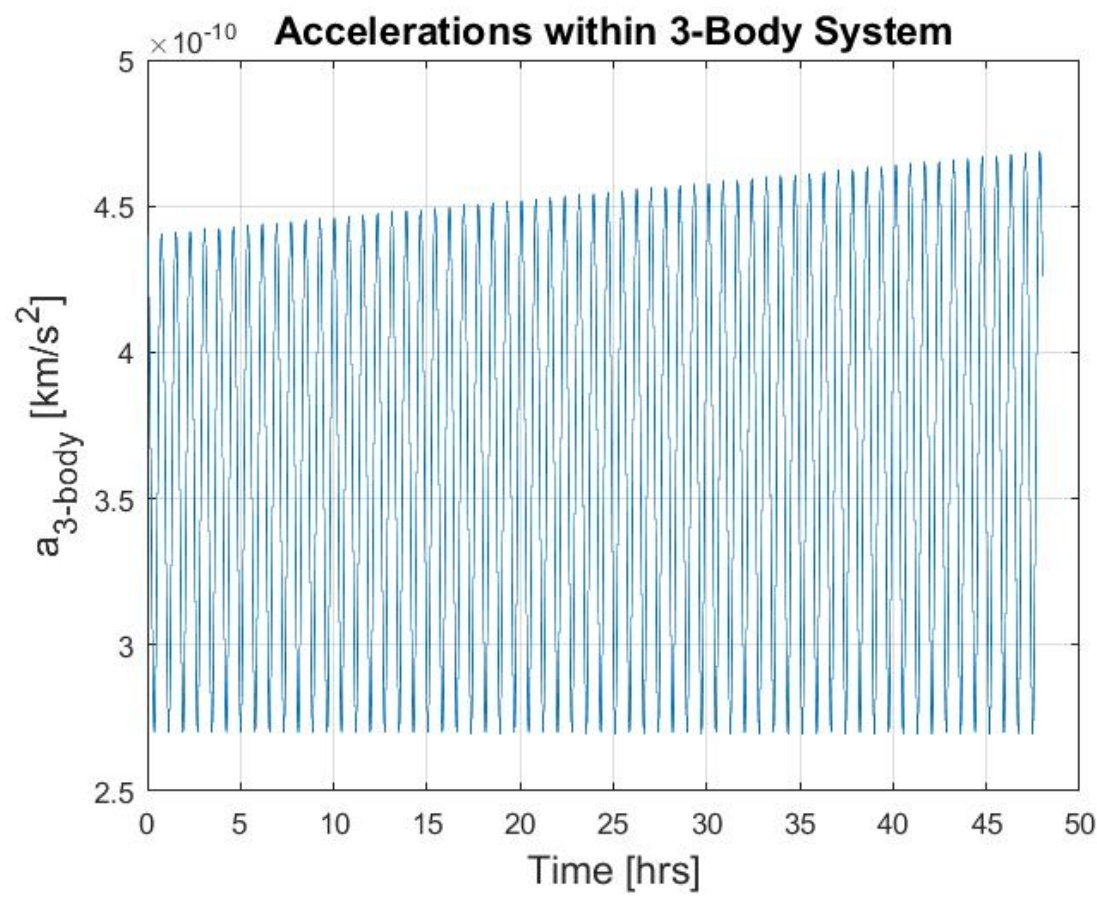

Figure 2.5: Spacecraft accelerations due to 3-body system over first two days of mission.

The magnitude of the 3-body perturbation is consistent with those observed by spacecraft at similar altitudes confirming the accuracy of the calculations. 


\subsection{Initial Conditions}

In order to verify the feasibility of a CubeSat mission involving formation flight, a concept of operations including the initial stages must be detailed. The formation flight mission was modeled to imitate the ISS NanoRack deployment capability[18]. Both 3U CubeSats were modeled to simultaneously leave the ISS with a low relative velocity to the station and an even lower relative velocity to each other. The ISS was assumed to have the capability to deploy the satellites into circular orbits at their altitude of roughly $405 \mathrm{~km}$. The initial orbital elements of both satellites are displayed in Table 2.2. To obtain these orbital elements, an arbitrary TLE from the ISS was obtained from March 28, 2018. This epoch was then designated as the time of simulated deployment from the NanoRacks aboard the ISS. This designation along with the assumption of a circularized deployment allows for the determination of the orbital elements in Table 2.2 .

Table 2.2: Initial satellite orbital elements.

\begin{tabular}{l|cccccc} 
Satellite & $a[\mathrm{~km}]$ & $e$ & $i\left[^{\circ}\right]$ & $\omega\left[^{\circ}\right]$ & $\Omega\left[^{\circ}\right]$ & $\nu\left[^{\circ}\right]$ \\
\hline 3U CubeSat & 6783.273 & $1.2991 \mathrm{e}-4$ & 51.6425 & 232.39 & 61.1386 & 269.992 \\
\hline
\end{tabular}

Both satellite states vary by decimals lower than stated above due to their simultaneous deployment. A variance in their semimajor axes of $\sim 1 \mathrm{e}-4$ indicates separation in their initial positions by a distance of approximately $30 \mathrm{~cm}$ as would be appropriate for the NanoRack initialization[19]. From this initial configuration, the simulation proceeds to control the satellite orientation to achieve a desired $1 \mathrm{~km}$ separation. The achievement and maintenance of this separation over time will validate the feasibility of the CubeSat formation flight mission. 


\subsubsection{NanoRack Deployment}

For this study, the scenario in which two $3 \mathrm{U}$ CubeSats are deployed simultaneously from the ISS NanoRack system. Although documentation is lacking for this deployment technique, several videos exists that capture what the process may entail[19]. From these videos, the initial conditions of satellite deployment were modeled to imitate what was filmed. As such, the two $3 \mathrm{U}$ CubeSats were modeled to be deployed simultaneously from the ISS at the designated epoch.

\subsubsection{Relative Velocity}

From observing film of NanoRack deployment, the CubeSats appear to have a small relative velocity to the ISS and even smaller relative velocities to each other as they drift off screen[19]. However, the magnitude of this relative velocity is uncertain. As stated, the CubeSats were assumed to have an initial relative position of $30 \mathrm{~cm}$. From this configuration, the satellites were assumed to drift in position by a few meters each orbit when uncontrolled. This situation is corroborated with what is observed in film[19].

\subsubsection{Orbit Circularization}

For the purpose of the mission, it is important that the CubeSats be injected into circular orbits. While the ISS already sits in a mostly circular orbit, it is unverified whether the NanoRacks deployment system can deliver the satellites into similar

circular orbits. With the low relative velocity between the ISS and the deployed satellites, it was assumed that the NanoRacks were able to maintain the circularized orbits of the CubeSats, allowing for the mission to proceed. 


\subsection{Model Constraints}

The most limiting factor in achieving a higher fidelity model is that of time. While running the model in the Simulink environment, the dynamics are integrated and propagated utilizing the built-in Matlab numerical solver ode45[20]. The solver employs a adaptive Runge-Kutta integration method represented by the Butcher tableau displayed in Fig. 2.6[20].

\begin{tabular}{l|llllll}
0 & & & & & \\
$1 / 4$ & $1 / 4$ & & & & & \\
$3 / 8$ & $3 / 32$ & $9 / 32$ & & & & \\
$12 / 13$ & $1932 / 2197$ & $-7200 / 2197$ & $7296 / 2197$ & & & \\
1 & $439 / 216$ & -8 & $3680 / 513$ & $-845 / 4104$ & & \\
$1 / 2$ & $-8 / 27$ & 2 & $-3544 / 2565$ & $1859 / 4104$ & $-11 / 40$ & \\
\hline & $16 / 135$ & 0 & $6656 / 12825$ & $28561 / 56430$ & $-9 / 50$ & $2 / 55$ \\
& $25 / 216$ & 0 & $1408 / 2565$ & $2197 / 4104$ & $-1 / 5$ & 0
\end{tabular}

\section{Figure 2.6: Finite differencing for adaptive Runge Kutta 45 method.}

The adaptive Runge-Kutta 45 method is different from the Runge-Kutta 4 method in that it has a 5th-order method for step size control [20], effectively changing the time step when the global error drifts farther than desired. While altering the step size when necessary provides a more accurate model, it can cause the simulation of a nonlinear system to slow down to an undesirable pace. This being the case, a higher fidelity model increases the nonlinearity of the spacecraft dynamics and causes the simulation to slow down until results cannot be produced at a reasonable pace. Two options exist to mitigate this problem. One option would be to use a lower order finite differencing method to propagate the equations of motion at the expense of accuracy. The second option would be to limit the level of fidelity of the model to effectively limit the time it takes to simulate the higher order solver. It is for this reason that the fidelity of some environmental parameters was sacrificed for the sake of time. 
Chapter 3

\section{SYSTEM CONTROL}

A key component to proper execution of the mission is the control law used for commanding orientation. With the control used, the magnitude of control torques necessary for these commands is compared against the torque available from the magnetorquer actuators. If the command torques necessary to execute maneuvers is less than the torque available from the actuators, then the feasibility of the mission can be confirmed.

\subsection{PD Control}

In order to command spacecraft orientation, a Proportional Derivative, or PD, controller is designed as shown in Equation 3.1[16] where $\epsilon_{e}$ is the error in spacecraft attitude from the commanded attitude represented in quaternion notation and $\omega_{e}$ is the error is spacecraft angular velocity from the desired angular velocity.

$$
T_{c}=-K_{p} \epsilon_{e}-K_{d} \omega_{e}
$$

The PD controller is analogous to a mass-spring-damper system. The proportional control acts as a spring with constant $K_{p}>0$ to scale the error signal[16]. However, solely proportional control results in undesirable undamped oscillatory motion constantly overshooting the desired attitude. Therefore, adding a derivative term $K_{d}>0$ will allow the system to settle on the commanded orientation. With a constant reference attitude, any oscillatory behavior will die out due to the damping term[16]. To implement the PD control, quaternion multiplication between the commanded quaternion conjugate, $q_{c}^{*}$ and the current spacecraft quaternion, $q$ is used to extract $\epsilon_{e}$ as shown in Equation 3.2. In addition, the error in angular velocity is shown in 
Equation 3.3 with $\omega$ being the spacecraft's current angular velocity and $\omega_{c}$ being the reference angular velocity.

$$
\begin{gathered}
q_{e}=q_{c}^{*} q=\eta_{e}+\epsilon_{e} \\
\omega_{e}=\omega-\omega_{c}
\end{gathered}
$$

As a mass-spring-damper system would exhibit a natural frequency, so does the spacecraft control behavior. To approximate the natural frequency of the system, a desired settling time and damping coefficient is assumed. The natural frequency is then approximated using Equation 3.4[16] where $t_{s}$ is the desired settling time and $\zeta$ is the damping coefficient of the system.

$$
\omega_{n} \approx \frac{4.4}{\zeta t_{s}}
$$

Once the natural frequency of the system is calculated, both proportional and derivative gains are determined using Equations 3.5[16] and 3.6[16] where $J$ is the inertia matrix of the spacecraft.

$$
\begin{gathered}
K_{p}=2 J \zeta \omega_{n} \\
K_{d}=2 J \omega_{n}^{2}
\end{gathered}
$$

For the mission being modeled, the assumed and calculated control parameters are displayed in Table 3.1. With a three dimensional non-symmetric spacecraft, different values are assigned for the inertia along each axis. For this reason, although the settling times and damping coefficients were chosen to be the same in all axes, the proportional and derivative gains vary between axes. In alternative simulations, settling times and damping coefficients can be altered to optimize gains for most effective control. For the purposes of this simulation, the consistent damping coefficient and settling time are sufficient for validation of initial results. 


\section{Table 3.1: Spacecraft control parameters.}

\begin{tabular}{l|cccccc} 
CubeSat Axis & $t_{s}[\mathrm{~s}]$ & $\zeta$ & $\omega_{n}[\mathrm{rad} / \mathrm{s}]$ & $J\left[\mathrm{~kg} \mathrm{~m}^{2}\right]$ & $K_{p}$ & $K_{d}$ \\
\hline X - longitudinal & 30 & 0.65 & 0.2256 & 0.0067 & $6.8 e-4$ & 0.0019 \\
Y - lateral & 30 & 0.65 & 0.2256 & 0.0417 & $3.39 e-3$ & 0.0097 \\
Z - lateral & 30 & 0.65 & 0.2256 & 0.0417 & $3.39 e-3$ & 0.0097 \\
\hline
\end{tabular}

\subsection{Timing Control}

For the mission being analyzed, open loop control is utilized for spacecraft orientation control. For implementation, a schedule is determined over the course of the mission to achieve the desired $1 \mathrm{~km}$ separation. In order to determine the desired schedule, several aspects of the mission were taken into account.

To begin the mission, the spacecraft would have left the ISS from the NanoRack deployment system. For the first few hours, it can be imagined that this time would be used for detumble, health checks, and subsystem monitoring. It is for this reason that the first four hours allow the spacecraft to fly passively, only maintaining their initial orientation.

After the first four hours, the spacecraft at a lower altitude (Spacecraft A) is commanded to turn its orientation so that the maximum drag configuration is experienced. This shift in orientation marks the initialization of the separation maneuver. Due to the fact that the desired separation distance is $1 \mathrm{~km}$, the two spacecraft are allowed to drift to a relative position of $500 \mathrm{~m}$. At this time, Spacecraft A returns its orientation to the minimum drag configuration while spacecraft B simultaneously alters its orientation to the maximum drag configuration. This maneuver marks the midway point of the spacecraft separation phase. The two spacecraft are held in these orientations for the second half of the separation phase until the two spacecraft have a relative position of $1 \mathrm{~km}$. 
Once the desired separation of $1 \mathrm{~km}$ is reached, spacecraft B returns its orientation to the minimum drag configuration so that both vehicles hold their longitudinal axes in the v-bar direction. For this final phase, the spacecraft are intended to hold their relative distance of $1 \mathrm{~km}$ for the remainder of the mission.

It was determined that the mission described will take just under 2 weeks to complete. The mission schedule is concisely laid out in Table 3.2.

Table 3.2: Mission schedule.

\begin{tabular}{l|ccc}
\multirow{2}{*}{ Time [days] } & Spacecraft A & Spacecraft B & Relative Distance [m] \\
\hline 0 & Min. Drag & Min. Drag & 0.3 \\
0.167 & Max. Drag & Min. Drag & 0.3 \\
5.208 & Min. Drag & Max. Drag & 500 \\
10.25 & Min. Drag & Min. Drag & 1000 \\
\hline
\end{tabular}


Chapter 4

RESULTS

After running the simulated two week mission, it was determined that the predetermined schedule successfully achieved a result of the $1 \mathrm{~km}$ separation between spacecraft. Several parameters were retrieved from the simulation and analyzed to assess the success of the mission.

\subsection{Control Verification}

To verify mission success, the attitudes of both spacecraft were tracked throughout the two week period and performance was assessed based on the scheduled maneuvers. In order to acquire the proper orientations, commanded quaternion components were determined for the maximum and minimum drag configurations. These quaternions used for attitute control throughout the mission are listed in Table 4.1.

Table 4.1: Quaternion Commands

\begin{tabular}{l|cccc} 
CubeSat Orientation & $\eta$ & $\epsilon_{1}$ & $\epsilon_{2}$ & $\epsilon_{3}$ \\
\hline Maximum Drag & 0.7071 & 0 & 0.7071 & 0 \\
Minimum Drag & 1 & 0 & 0 & 0 \\
\hline
\end{tabular}

Over the course of the mission, both spacecraft A and spacecraft B executed maneuvers to change their attitude to either configurations. In order to verify these maneuvers were executed correctly, the quaternion components of the spacecraft were tracked during the simulation to ensure the commanded quaternions were met. The scheduled maneuvers at 4 hours, 5.208 days and 10.25 days can be identified in Fig. 4.1 where the corresponding quaternion components of each CubeSat are altered 
indicating a change in attitude.
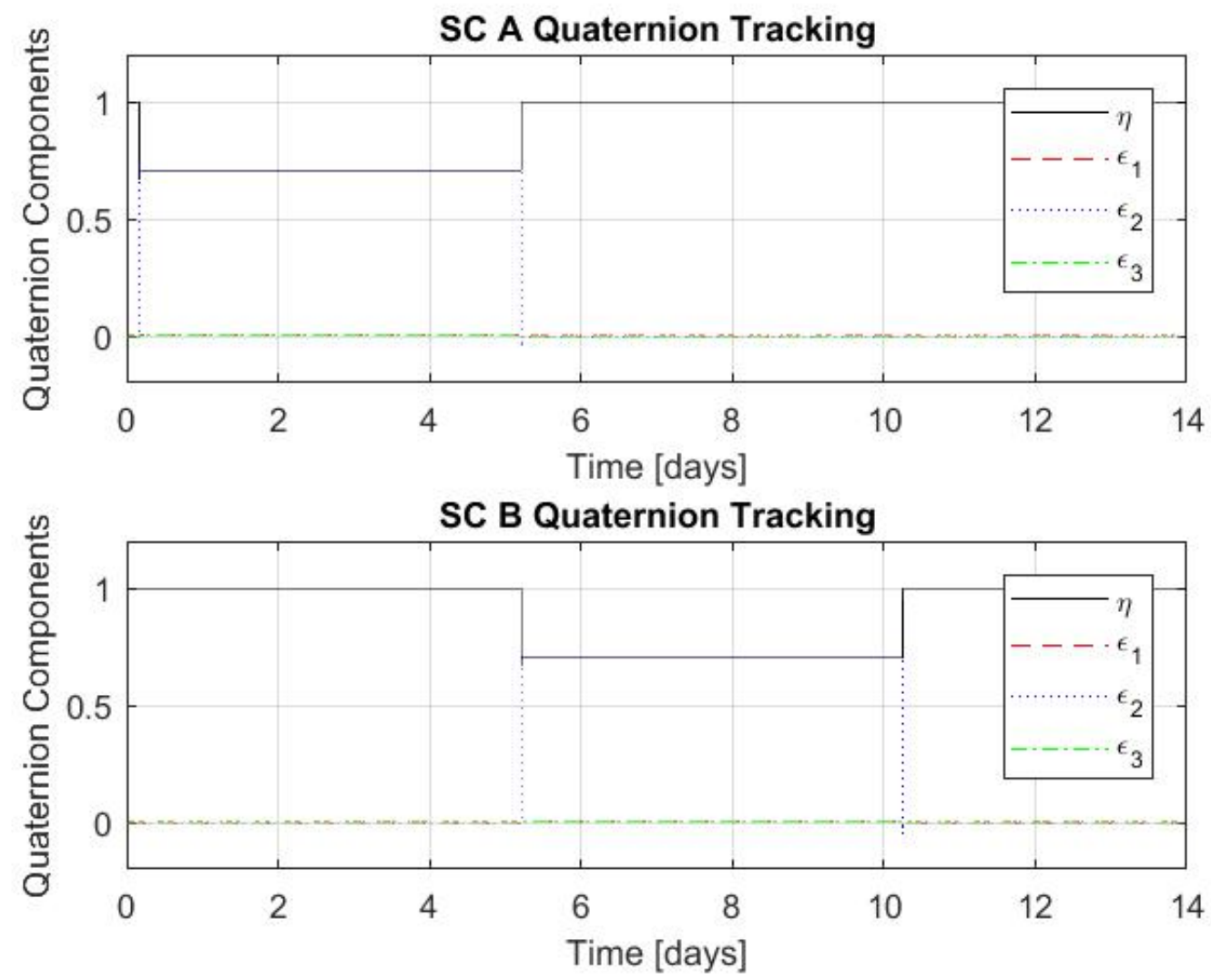

Figure 4.1: Quaternion components over mission lifetime.

As commanded, spacecraft $\mathrm{A}$ is the first spacecraft to alter its orientation to be experiencing the maximum drag for a period of 5 days. After these first few days, spacecraft A returns to the minimum drag configuration while spacecraft B alters its orientation to experience maximum drag. After another 5 days, spacecraft B returns to the minimum drag configuration to maintain its relative position with spacecraft A. All maneuvers are identifiable in Fig. 4.1 and verified to be executed as planned. 


\subsection{Spacecraft Altitude}

With the deployment from the ISS NanoRacks, the CubeSats have an initial altitude of $405 \mathrm{~km}$. However, Earth's oblateness causes the altitude of both spacecraft to vary periodically, as shown in Fig. 4.2.

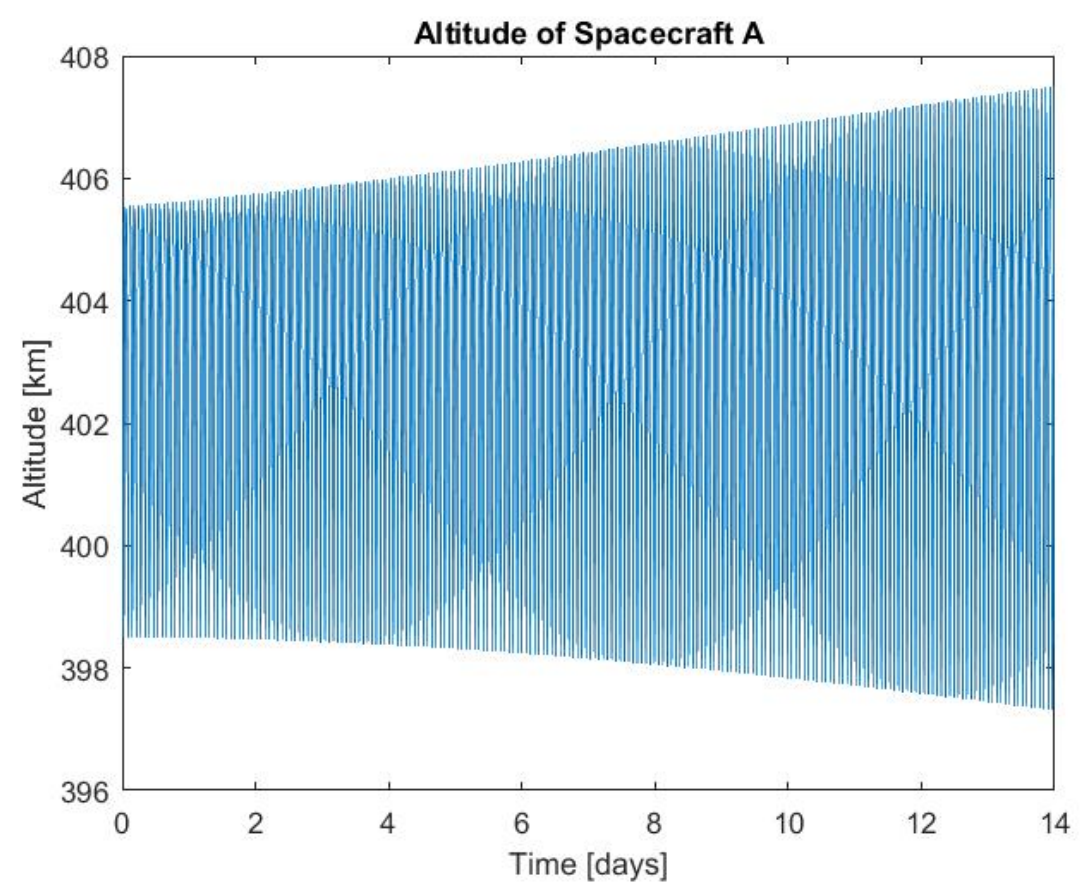

Figure 4.2: Altitude of spacecraft A over mission lifetime.

As predicted, the lower bound of the altitude drops as the spacecraft continually experiences the drag force of the atmosphere. A unique quality of the effect of Earth's oblate nature is that it will tend to increase the eccentricity of a spacecraft's orbit if it is not accounted for with stationkeeping. This quality is also illustrated by Fig. 4.2. It is for this reason that although the lower bound of the spacecraft altitude decreases, the upper bound of the altitude increases as well, described by an increase in eccentricity of the orbit. This behavior is also experienced with the altitude of spacecraft B. 


\subsection{Relative Position}

Being the focus of the mission objective, relative position of the spacecraft was recorded over time. While the altitude of both spacecraft tends to fluctuate due to Earth's oblateness, their relative position is not affected by this behavior as they both experience it similarly and at relatively the same time. It is for this reason that only small fluctuations of relative position are noticeable. The relative position of the spacecraft over the lifetime of the mission is displayed in Fig. 4.3.

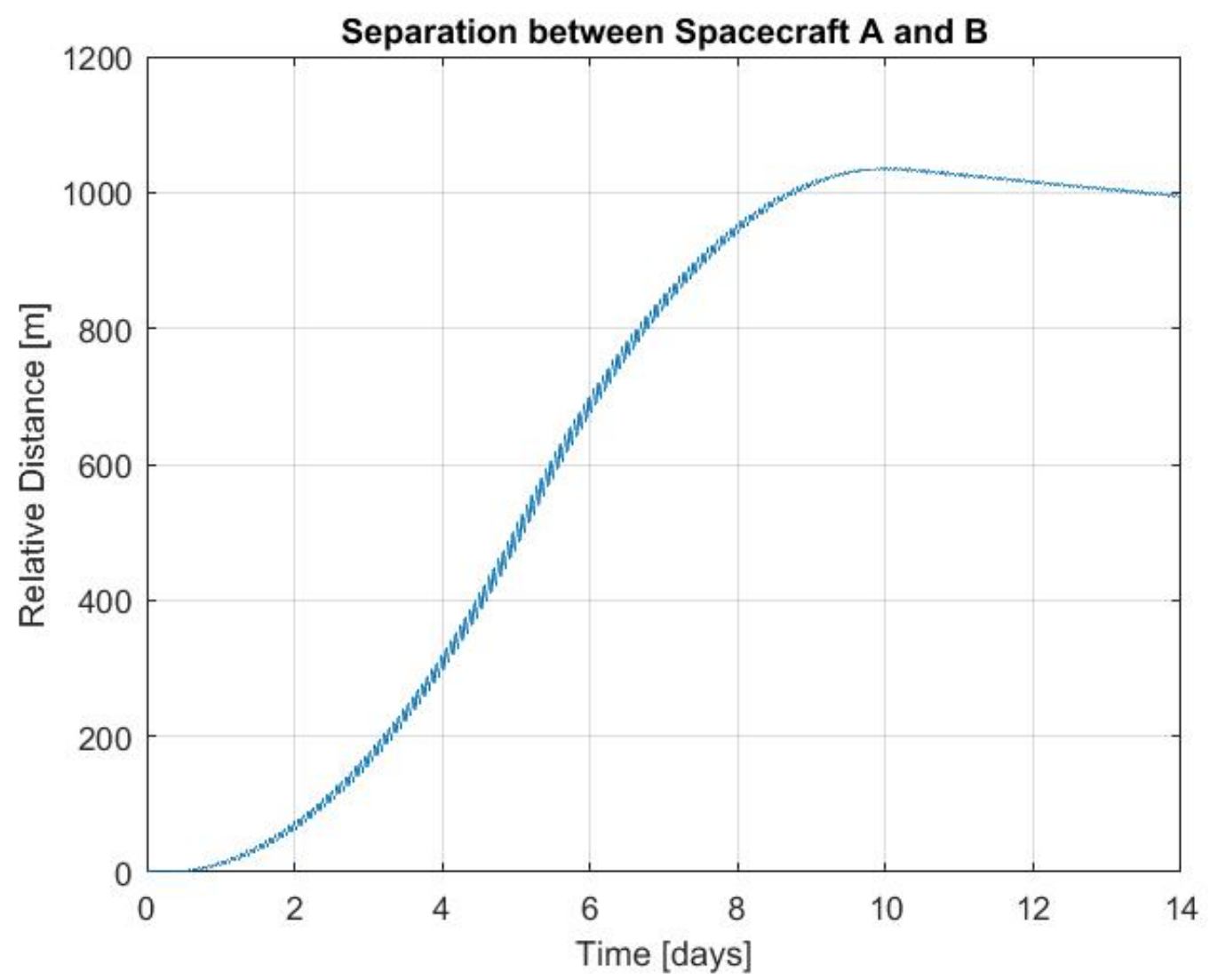

Figure 4.3: Relative position of spacecraft over mission lifetime.

With all maneuvers executed correctly, the two spacecraft are able to achieve and maintain a relative distance of $1 \mathrm{~km}$ over the course of the 2 week mission. The success of the simulation again reinforces the idea of mission feasibility. 


\subsection{Control Torques}

In order to further verify mission feasibility, the magnitude of the control torques required was determined over the course of the mission. the control torques necessary to carry out the mission are displayed in Fig. 4.4.
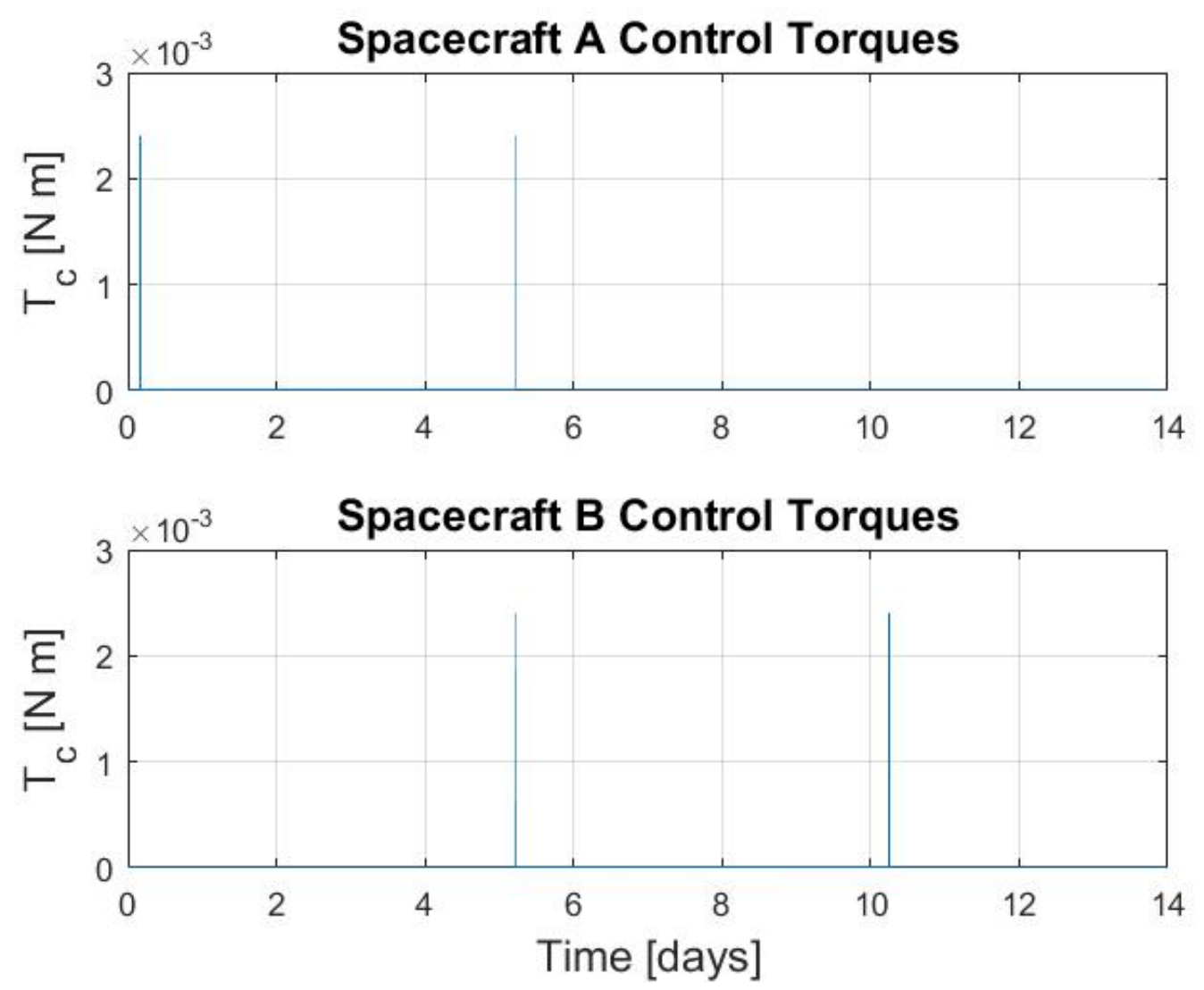

Figure 4.4: Spacecraft control torques over mission lifetime.

At first glance, the most notable aspect of the plot are the spikes in torque magnitude. These spikes indicate a spacecraft maneuver. From previous determination, the ISIS Magnetroquer Board discussed will only allow for the application of $\sim 5 e-6$ Nm of torque. While this may seem discouraging, paths of mitigating these spikes in torque magnitude will be discussed momentarily.

More importantly are the torques necessary for maintaining a commanded ori- 
entation during the longer phases of the mission. During these phases, disturbance torques including drag, gravity gradient, and SRP are modeled. In order to maintain the necessary orientations, both satellites must use their magnetorquer boards to combat these disturbances. The magnitude of the required control torque to mitigate these disturbances is displayed in Fig. 4.5.

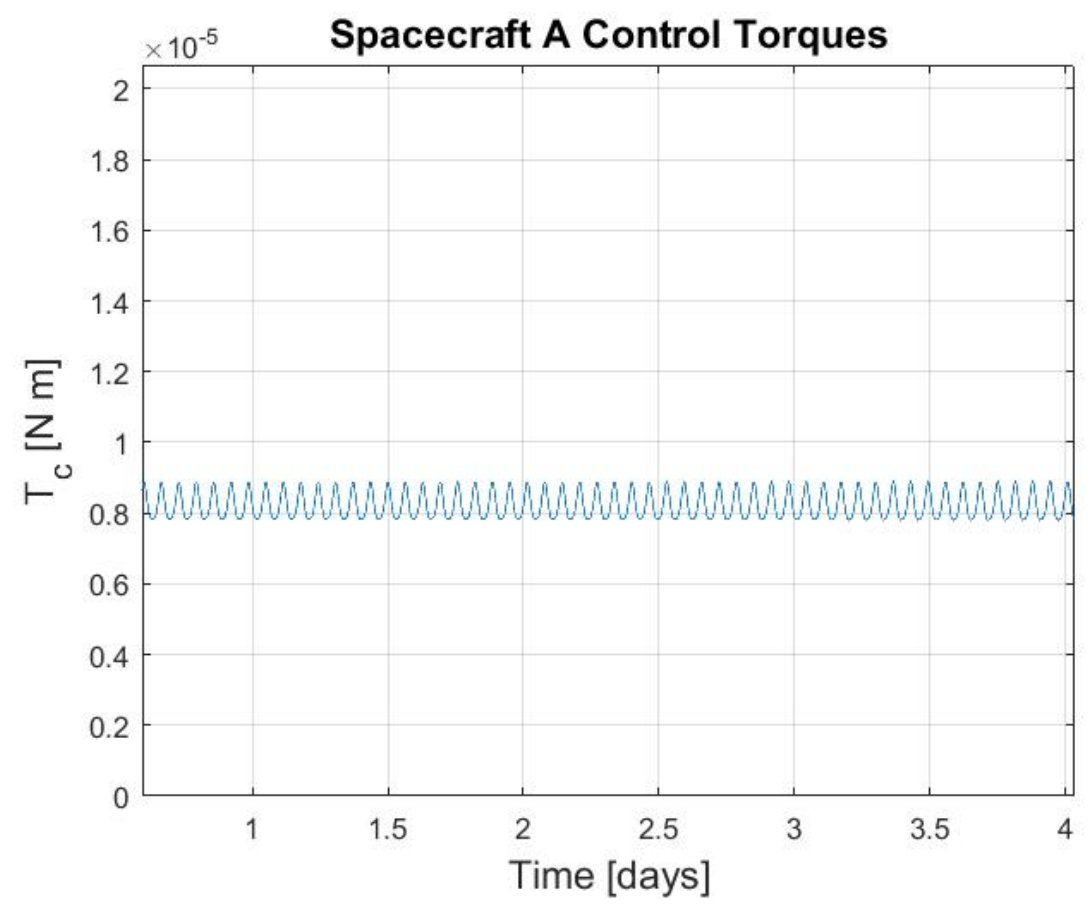

Figure 4.5: Spacecraft control torques during nominal phase.

As determined from the plot, the required torque for maintaining a spacecraft attitude is $\sim 1 e-5$ which is slightly above the available torque.

In order to rectify this glaring issue, attention was turned towards the control law implementation. As displayed in Table 3.1, a settling time of 30 seconds was used for control. While this provided apt orientation control, this response is not consistent with electromagnetic actuation as it is implemented in a much more extended process. For this reason, a new settling time of 15 minutes was chosen to better reflect a space mission application. The simulation was run again with the spacecraft control 
parameters as shown in Table 4.2 and mission schedule displayed in Table 4.3.

Table 4.2: Alternative spacecraft control parameters.

\begin{tabular}{l|cccccc} 
CubeSat Axis & $t_{s}[\mathrm{~min}]$ & $\zeta$ & $\omega_{n}[\mathrm{rad} / \mathrm{s}]$ & $J\left[\mathrm{~kg} \mathrm{~m}^{2}\right]$ & $K_{p}$ & $K_{d}$ \\
\hline X - longitudinal & 15 & 0.65 & 0.00752 & 0.0067 & $7.54 e-7$ & $6.51 e-5$ \\
Y - lateral & 15 & 0.65 & 0.00752 & 0.0333 & $3.77 e-6$ & $3.26 e-4$ \\
Z - lateral & 15 & 0.65 & 0.00752 & 0.0333 & $3.77 e-6$ & $3.26 e-4$ \\
\hline
\end{tabular}

Table 4.3: Alternative mission schedule.

\begin{tabular}{l|ccc} 
Time [days] & Spacecraft A & Spacecraft B & Relative Distance $[\mathrm{m}]$ \\
\hline 0 & Min. Drag & Min. Drag & 0.3 \\
0.167 & Max. Drag & Min. Drag & 0.3 \\
5.208 & Min. Drag & Max. Drag & 500 \\
10.25 & Min. Drag & Min. Drag & 1000 \\
\hline
\end{tabular}

With this new simulation, all parameters were tracked just as they were previously. To verify the mission was just as successful as the prior mission architecture, the relative distance of the satellites is displayed in Fig. 4.6. Here it is shown that the satellites were still able to achieve a relative distance of $1 \mathrm{~km}$ and hold the spacing for the remainder of the two week mission.

While the relative position is a good indicator for whether the desired mission outcome was achieved, the control torques and resultant quaternion components from the alternative control implementation are more valuable in terms of determining mission feasibility. These results were tracked throughout the simulation and are displayed in Fig. 4.7 and Fig. 4.8

From these figures, the reduction in control torque magnitude is apparent. In order to perform the maneuvers necessary for the mission, the control scheme never requires torques greater than $4 e-6 \mathrm{Nm}$, well within the range of COTS magnetor- 


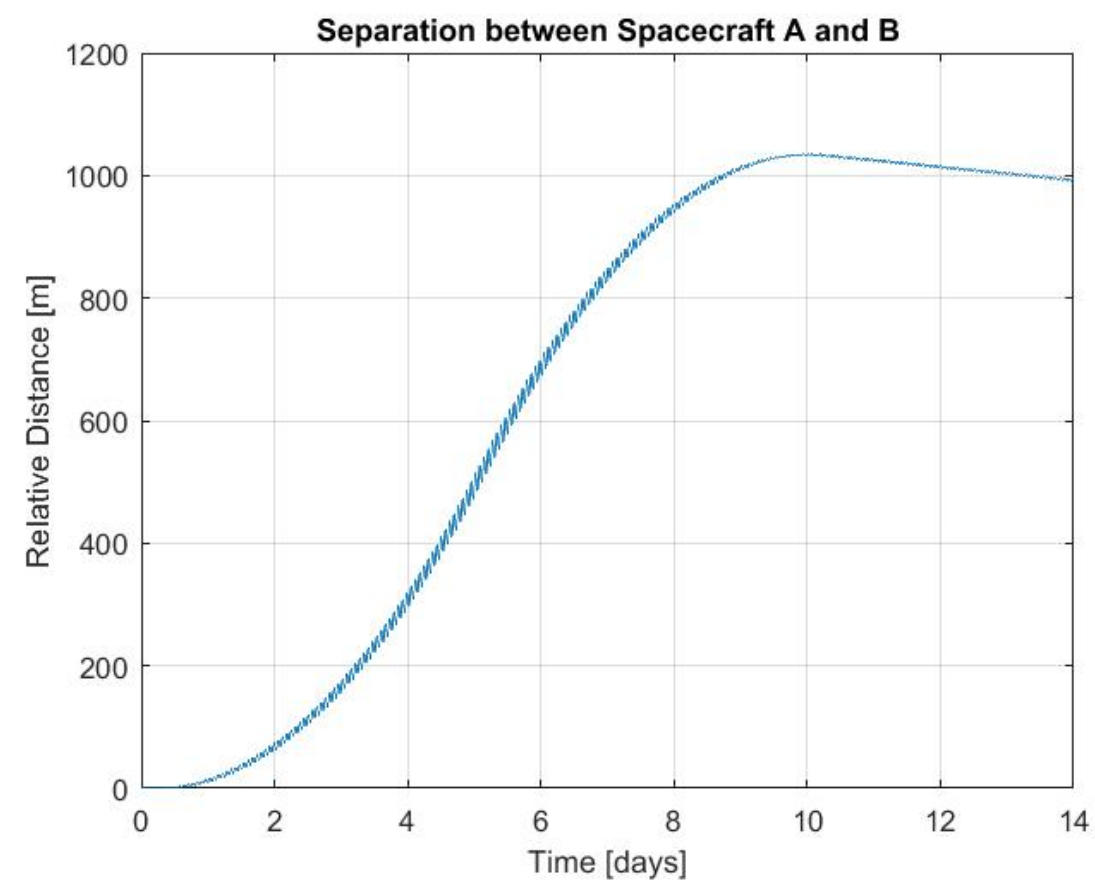

Figure 4.6: Relative position of spacecraft for alternative mission.
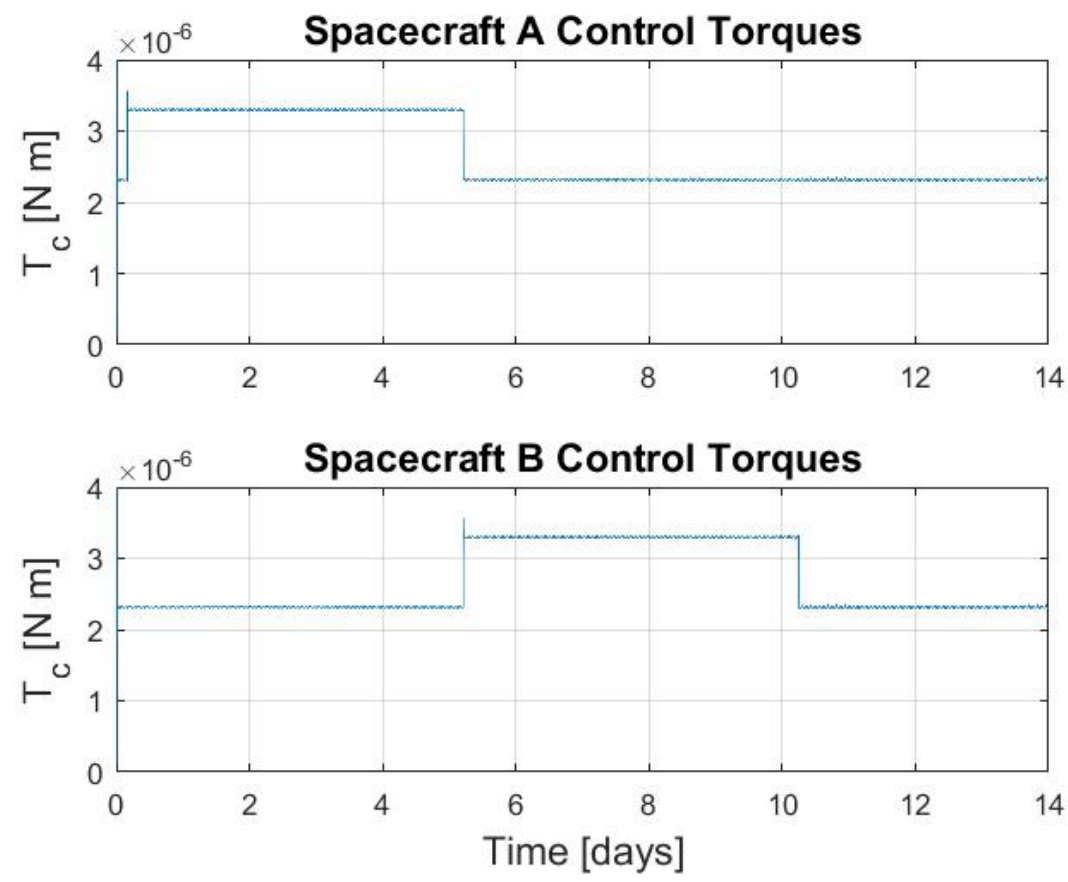

Figure 4.7: Control torque magnitudes for alternative mission. 

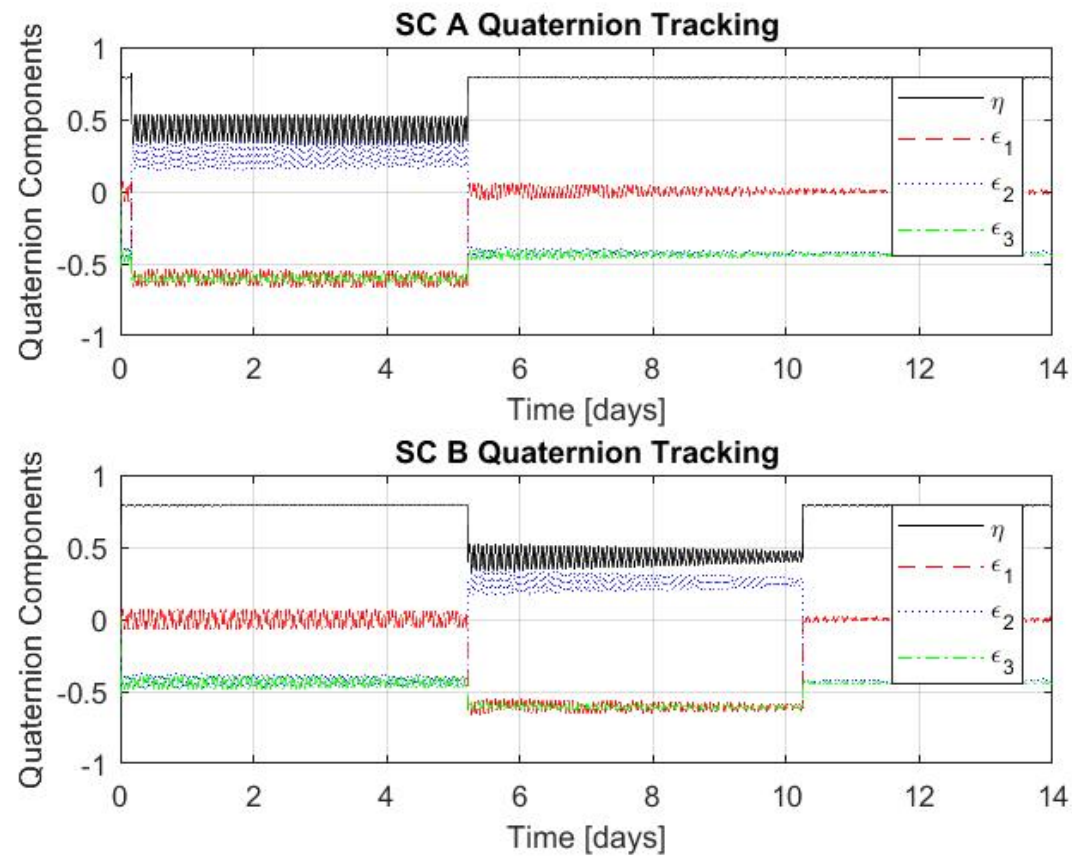

\section{Figure 4.8: Quaternion components for alternative mission.}

quer boards such as the ISIS board. However, a less than ideal result is apparent when viewing the quaternion components over the course of the alternative mission. With a settling time of 30 seconds, the spacecraft is able to maintain the desired orientations without much variance whereas the settling time of 15 minutes causes both satellites to oscillate around the desired orientations for each command. This oscillation causes some assumptions in the simulation to breakdown and may or may not be crucial in determining mission success or failure in a space application. In the model, it is assumed that the spacecraft maintain the incident surface areas associated with the maximum and minimum drag configurations when either spacecraft is commanded to the desired orientation. With a shorter settling time this assumption was valid. Now, with the introduction of a longer settling time and the observed oscillation that accompanies it, the incident surface area will vary based on the slightly correcting and re-correcting spacecraft orientation during the commanded schedule. When commanding a minimum drag configuration, the spacecraft will expose a larger 
wetted area than what is simulated. Similarly, when commanding the maximum drag configuration, the spacecraft will expose a smaller wetted area than indicated due to the oscillation. This behavior is indicative of lower fidelity physics within the model and raises concern. Depending on the application, this lack of pointing control could prove troublesome for mission success. In order to model this behavior properly, the wetted area incident to the v-bar direction would be calculated at each time step and fed back into the spacecraft dynamics. Without this higher fidelity modeling, it is not possible to tell whether this alteration in spacecraft performance is critical to mission success. Although this behavior raises concerns, the overarching reduction in control torque magnitudes is a major indicator of mission feasibility.

\subsection{Alternative Solutions}

For the entirety of this study, the control torques required by the mission have been compared to what is available from the ISIS magnetorquer board as it is a good representation of a COTS electromagnetic actuation board. However, alternative solutions exist. Firstly, while it is widely accepted that $0.2 \mathrm{Am}^{2}$ is the upper limit of magnetorquer performance, some sources such as the University of Michigan boast a higher performance. In this instance, Michigan claims a performance of $0.36 \mathrm{Am}^{2}$ with their specially designed CADRE coils[11]. Operating at $400 \mathrm{~km}$ with a magnetic field of approximately 26,000 $n T$, this would allow for a control torque of $\sim 9 e-6 \mathrm{Nm}$. This capability, if made readily available, would allow for the reduction of settling time for control and mitigation of the observed oscillations.

However, this proposed solution still implies a spacecraft under the sole influence of electromagnetic actuation. There are COTS units for CubeSats such as the Maryland Aerospace Incorporated 400 Mini ADACS Unit that incorporate both magnetorquers and reaction wheels to provided larger torque than a single actuator would apply 
independently[2]. With the addition of reaction wheels, the ADACS can provide torques up to $6 e-3 \mathrm{Nm}[2]$, a substantial jump from the ISIS board. The 400 Mini ADACS Unit is pictured in Fig. 4.9.

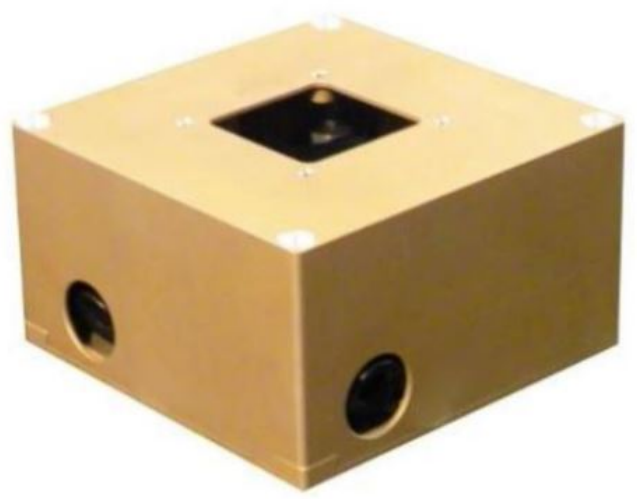

Figure 4.9: MAI 400 Miniature Attitude Determination and Control System.[2]

If a unit such as this were implemented, a propulsionless microsatellite mission would still be realized and the upper bound of control torque magnitude would be increased greatly. Mission complexity and cost would be raised, but whether that proves troublesome would be mission specific. 
Chapter 5

CONCLUSIONS

With the medium to high fidelity model being implemented, the feasibility of the simulated mission was assessed. Incorporating higher order perturbational effects, spacecraft dynamics and control were determined. With the data retrieved from two simulation of varying control implementation, results were analyzed and logged.

\subsection{Discussion}

From the simulated results, feasibility is confirmed for the $3 \mathrm{U}$ CubeSat differential drag mission. However, some caveats exist surrounding this conclusion highlighting the limitations of the simulation and subsequent concerns. Some assumptions made and exterior considerations must be brought to light that may affect the application of this mission in the space environment.

\subsubsection{Spacecraft Characteristics}

The schedule for the simulated mission and the resulting time required for the desired satellite separation stems from the assumed spacecraft characteristics. Table 5.1 displays these characteristics.

With this geometry and mass previously defined, the areas incident during each mission phase can be calculated as well as the resulting area to mass ratio. These values are displayed in Table 5.2.

By altering the spacecraft geometry and as a result, the incident areas, the mission profile will be altered. Higher fidelity models should incorporate more complex spacecraft geometry as well as more accurate mass estimates to correctly model spacecraft 


\section{Table 5.1: Sapcecraft Characteristics}

\begin{tabular}{l|c} 
Characterstic & Assumed Value \\
\hline x-axis & $0.3[\mathrm{~m}]$ \\
y-axis & $0.1[\mathrm{~m}]$ \\
z-axis & $0.1[\mathrm{~m}]$ \\
Mass & $4[\mathrm{~kg}]$ \\
\hline
\end{tabular}

Table 5.2: Sapcecraft Characteristics

\begin{tabular}{l|cc} 
Spacecraft Orientation & Incident Area $\left[\mathrm{m}^{2}\right]$ & $\frac{A}{m}\left[\mathrm{~m}^{2} / \mathrm{kg}\right]$ \\
\hline Maximum Drag & 0.03 & 0.0075 \\
Minimum Drag & 0.01 & 0.0025 \\
\hline
\end{tabular}

behavior.

\subsubsection{Control Torques}

While the primary focus of this study was to determine the capabilities of electromagnetic actuation paired with differential drag and the feasibility of its application on a CubeSat mission, some alternative solutions were not assessed that still may lead to the overarching goal of propulsionless microsatellite formation flight. These alternatives include investigating stronger magnetorquer designs as well as integrated magnetorquer and reaction wheel systems. The incorporation of these solutions may provide further confirmation of mission feasibility.

\subsubsection{Detumble}

One phase that was neglected from the simulated mission is detumble. Both spacecraft are assumed to be deployed from the ISS NanoRacks with the desired initial orientation and no angular velocity. From the film studied, this is not an accurate 
representation of the deployment method. A more accurate model would incorporate a low angular velocity at the beginning of life. The CubeSats would then have to correct their angular rates using their magnetorquer boards and implementing what is known as B-dot control. B-dot control is a simple but powerful way to detumble spacecraft. The control law works by applying torque in the opposing direction that matches the rate of change of the magnetic field[2]. In order to incorporate this detumble regime, Earth's magnetic field and its directionality would be calculated at each time step, greatly increasing the fidelity of the model and its computational expense consequently. To better determine feasibility, detumble would be simulated and the control torques would be tracked and compared with the available torque.

\subsubsection{Perturbations}

With the medium to high fidelity model generated, the orbital perturbations mark periodic trends in the spacecraft positions and altitudes that cannot be mitigated by traditional stationkeeping methods. It is necessary to determine whether these periodic trends are disconcerting or if they may be ignored. By looking at these trends in relative position over the mission lifetime, one may be able to determine if the periodicity of distance proves to be mission threatening. For analyzing this periodicity, the final phase of the simulated mission in which the two spacecraft attempt to maintain a fixed relative distance is shown in Fig. 5.1.

The first trend to notice is the rapid periodic movement over the course of each orbit. The spacecraft tend to vary in relative distance by a few meters during each revolution. The determination of whether this behavior is mission threatening is mission specific and cannot be discussed relevantly within this context. The downward trend that is also exhibited by the CubeSats is not a result of orbital perturbations but rather the result of inaccurate final positioning. If both spacecraft were to end 


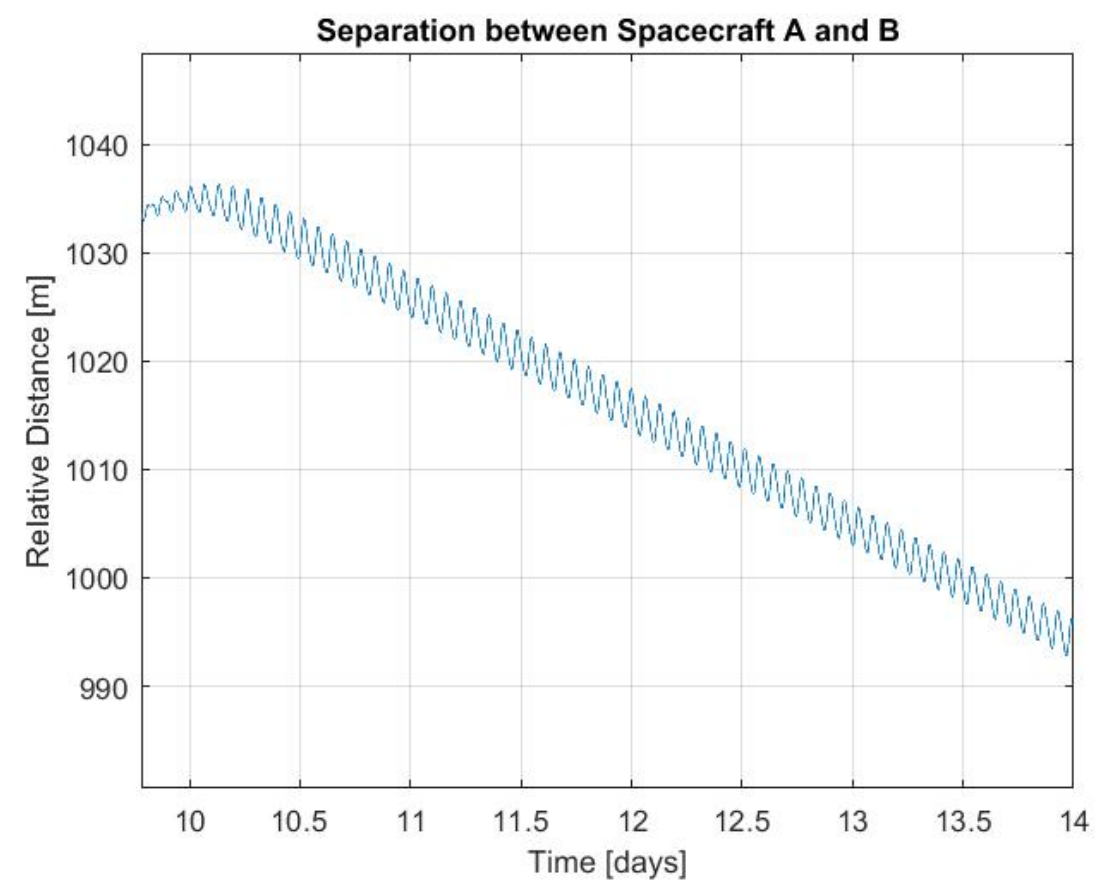

Figure 5.1: Periodicity in relative position.

the mission in the same orbit, this trend would not exist. A more strenuous control scheme may allow for this to be achieved.

\subsection{Future Work}

For continuing the study put forth by this thesis, the main goal is to increase the fidelity of the model created. While the model stands with medium to high fidelity, several aspects must be included to determine feasibility with absolute confidence. The simulation of the 2 week mission requires a 45 minute time span to compute. This being noted, a higher fidelity model will require more time. Things to be included in future simulations:

- Detumble and B-dot control implementation.

- Optimized closed loop control law. 
- Well defined spacecraft characteristics.

For the detumble regime, it will be necessary to determine what the magnitude of control torques will be and if they can be supplied by a COTS magnetorquer board with the performance of $0.2 \mathrm{Am}^{2}$. An optimized control law will allow for defining a mission schedule for increased spacecraft performance. In addition, the use of closed loop control will allow both spacecraft to enter the same final orbit, enabling efficient maintenance of relative position after the desired separation is achieved. Finally, a more well defined mission will determine realistic spacecraft characteristics for simulation. Spacecraft mass and geometry will determine the mission profile and overall performance. 


\section{BIBLIOGRAPHY}

[1] Inovative Solutions in Space, 2018. https://www.isispace.nl/product/isis-magnetorquer-board/.

[2] Meghan Quadrino, David Miller, and Kerri Cahoy. Testing the Attitude Determination and Control of a CubeSat with Hardware-in-the-Loop. Master's thesis, Massachusetts Institute of Technology, June 2014.

[3] Daniel W. Kwon. CubeSat Formation Control Using Differential Drag. Master's thesis, Massachusetts Institute of Technology, 2005.

[4] C. Escoubet, M. Fehringer, and M. Goldstein. The Cluster Mission. Annales Geophysicae, 19:1197-1200, 2001.

[5] Daniel Schulze, Manfred Zink, Gerhard Krieger, J. Ber, and Alberto Moreira. tanDEM-X Mission Concept and Status. Technical report.

[6] Michael R. LaPointe. Formation Flying with Shepherd Satellites. Technical report, December 2001.

[7] Emilien Fabacher, Stephanie Lizy-Destrez, Daniel Alazard, Finn Ankersen, and Jean-Francois Jourdas. Guidance and Navigation for Electromagnetic Formation Flight Orbit Modification. Technical report, April 2015.

[8] Sanny Omar. Using Differential Aerodynamic Forces for CubeSat Orbit Control. Auburn University Journal of Undergraduate Scholarship, 2014.

[9] A. Craig Stickler and K. T. Alfriend. Elementary Magnetic Attitude Control System. Journal of Spacecraft and Rockets, 13(5):282-287, 1976. 
[10] Peter J. Camillo and F. L. Markley. Orbit-Averaged Behavior of Magnetic Control Laws for Momentum Unloading. Journal of Guidance, Control, and Dynamics, 3(6):563-568, 1980.

[11] Duncan Miller. Toils for Coils, May 2013. https://www . aerospades . com/uploads/3/7/3/2/37325123/mxl_talk__toils_for_coils.pdf.

[12] Magnetic Field Calculators, 2018. https://www.ngdc.noaa.gov/geomag-web/\#igrfwmm.

[13] Howard D. Curtis. Orbital Mechanics for Engineering Students. Elsevier, 3rd edition, 2014.

[14] Hanspeter Shaub, Srinivas Vadali, John Junkins, and Kyle Alfriend. Spacecraft Formation Flying Control Using Mean Orbital Elements. Journal of the Astronautical Sciences, 48(1):69-87, 2000.

[15] Sanny Omar. CubeSat Formation Control Using Differential Drag. Master's thesis, Auburn University, 1513 Robertson Dr., Crestwood, KY 40014, 2014.

[16] Anton de Ruiter, Christopher Demaren, and James Forbes. Spacecraft Dynamics and Control: an introduction. Wiley, 1st edition, 2013.

[17] MathWorks, 2018. https:

//www.mathworks.com/products/matlab.html?s_tid=hp_products_matlab.

[18] NanoRacks LLC. NanoRacks CubeSat Deployer (NRCSD) Interface Control Document. Technical report, December 2013.

[19] NanoRacks. NanoRacks CubeSat Deployer Program-1 releasing satellites from the ISS, May 2013. https: //www youtube. com/watch?time_continue=4\&v=JQy9EwMrILI. 
[20] Numerical Integration of Differential Equations, 2018. https : //www . mathworks . com/help/matlab/examples/numericalintegration-of-differential-equations.html.

[21] W. de Vries. CubeSat Drag Calculations. Technical report, September 2010.

[22] Sanny Omar. Using Differential Aerodynamic Forces for CubeSat Orbit Control. Master's thesis, Auburn University, 1513 Robertson Dr., Crestwood, KY 40014, 2017.

[23] TDX (TanDEM-X: TerraSAR-X add-on for Digital Elevation Measurement), 2002. https://directory . eoportal .org/web/eoportal/satellitemissions/t/tandem-x.

[24] Satellite Drag, 2018. https://ccmc.gsfc.nasa.gov/RoR_WWW/SWREDI/2015/ SatDrag_YZheng_060415.pdf.

[25] Raymond Sedwick Alvar Saenz-Otero, Simon Nolet and David Miller. Developing and Maturing Micro-Satellite Formation Flight Technology. Technical report.

[26] Soon-Jo Chung. Design, Implementation and Control of a Sparse Aperture Imaging Satellite. Master's thesis, Massachusetts Institute of Technology, 2002.

[27] S. K. Shrivastava. Effects of Solar Radiation Pressure and Aerodynamic Forces on Satellite Attitude Dynamics and their Utilization for Control: a Survey. Technical report, July 1976.

[28] Li Qiao, Chris Rizos, and Andrew Dempster. Analysis and Comparison of CubeSat Lifetime. Technical report.

[29] Gill Montenbruck, Oliver and Eberhard. Satellite Orbits. Models, Methods, and Applications. Springer, 1st edition, 2000. 
[30] Frank Bauer John Bristow and Jonathan How. Enabling Spacecraft Formation Flying through Position Determination, Control, and Enhanced Automation Technologies. Technical report.

[31] Duncan Miller. Design optimization on the CADRE Magnetorquers. Technical report, May 2013. 


\section{APPENDICES}

Appendix A

COMPLETE RESULTS FROM PRIMARY SIMULATION

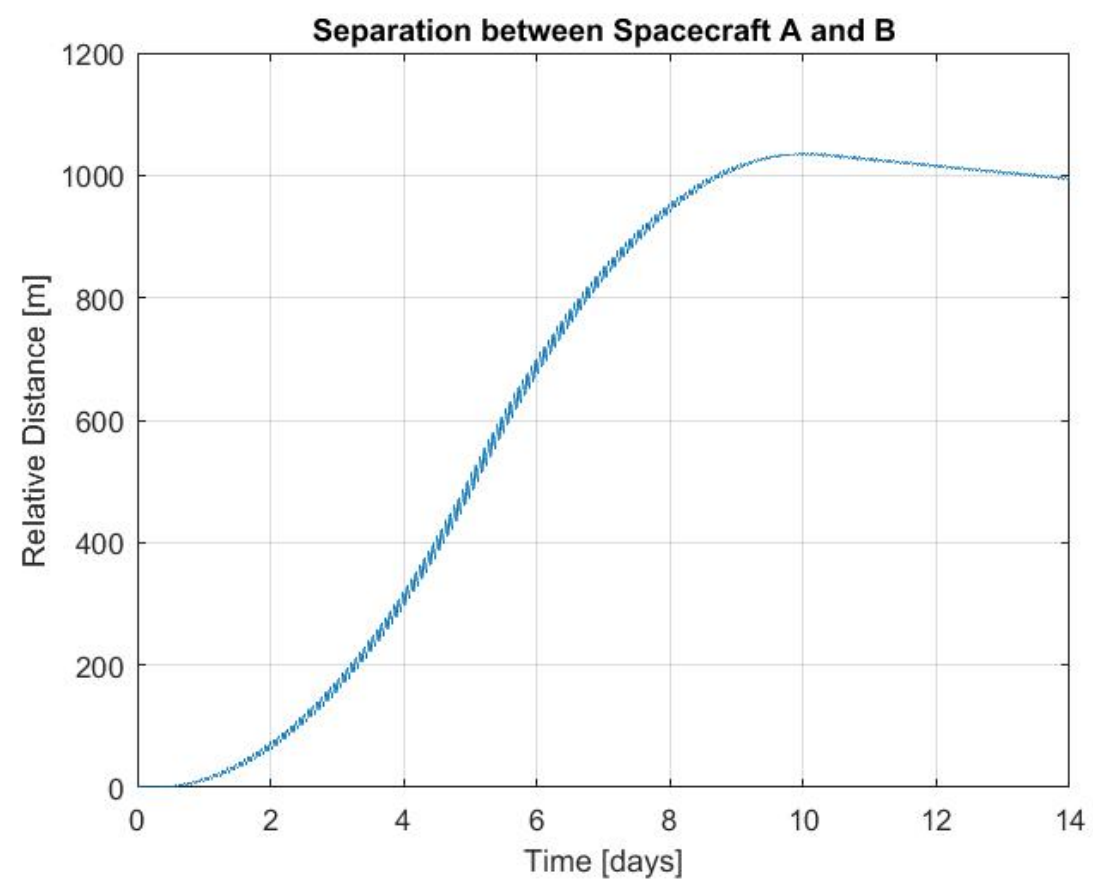

Figure A.1: Relative position of spacecraft over mission lifetime. 


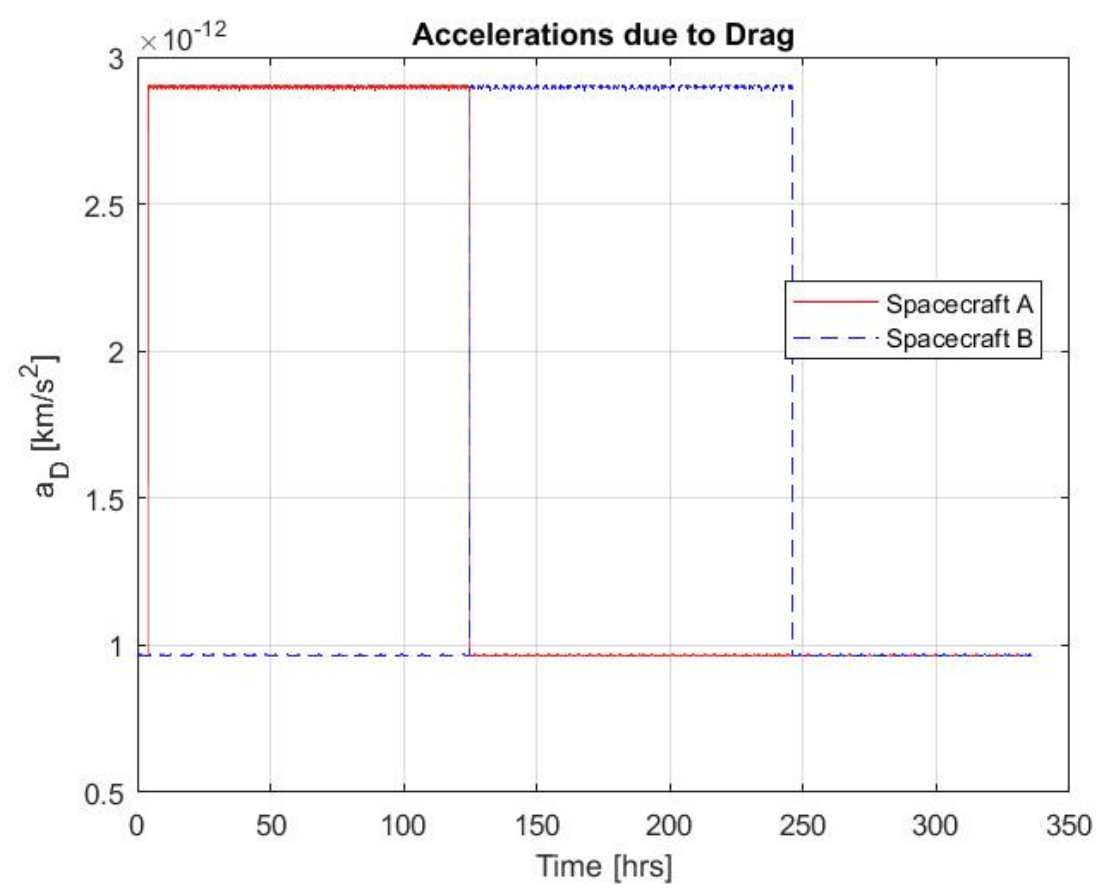

Figure A.2: Accelerations due to drag over mission lifetime.
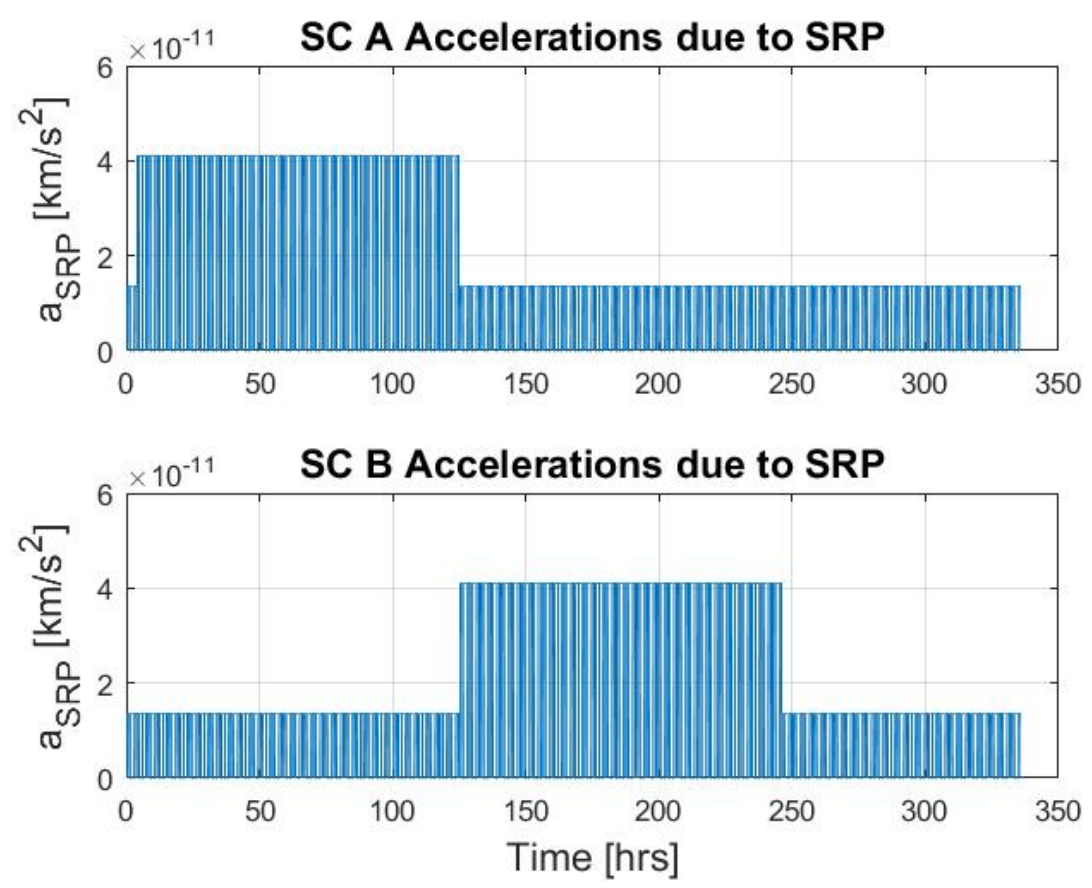

Figure A.3: Accelerations due to SRP over mission lifetime. 


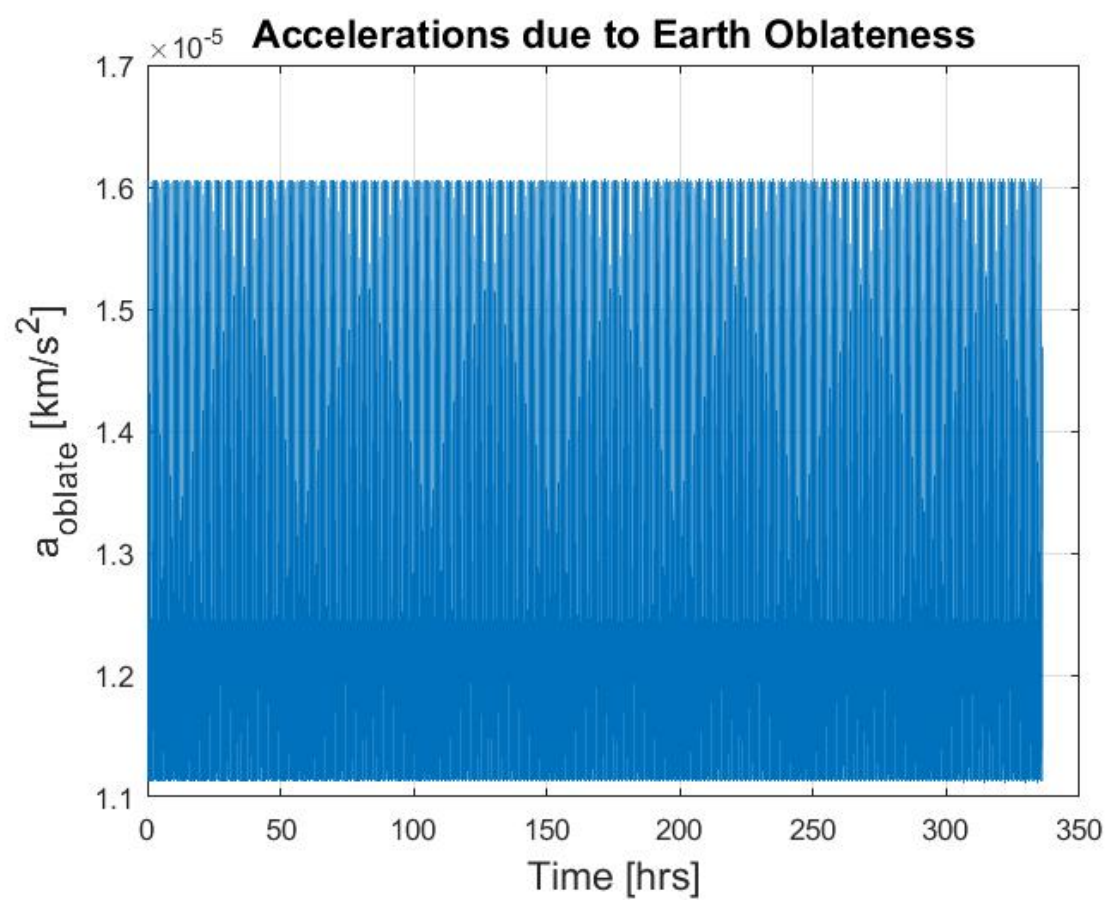

Figure A.4: Accelerations due to Earth oblateness over mission lifetime.

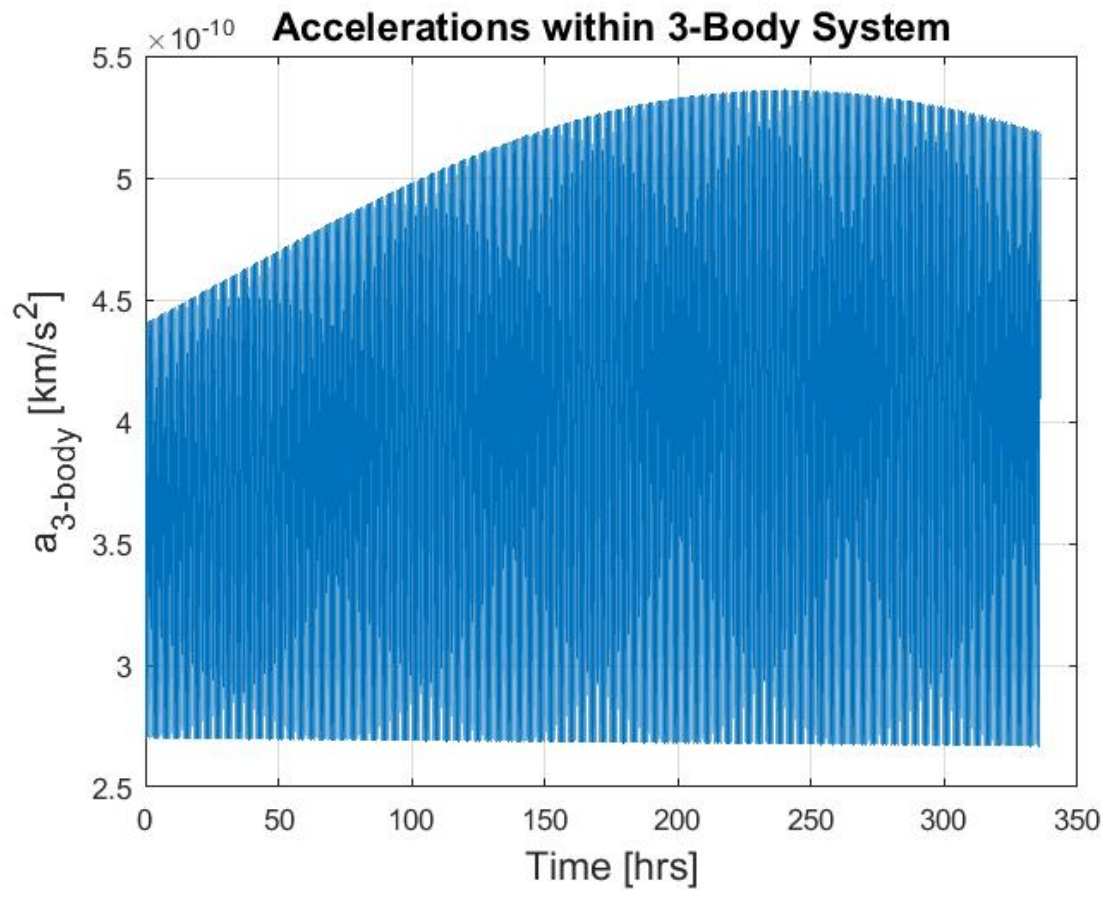

Figure A.5: Accelerations due to 3-body system over mission lifetime. 

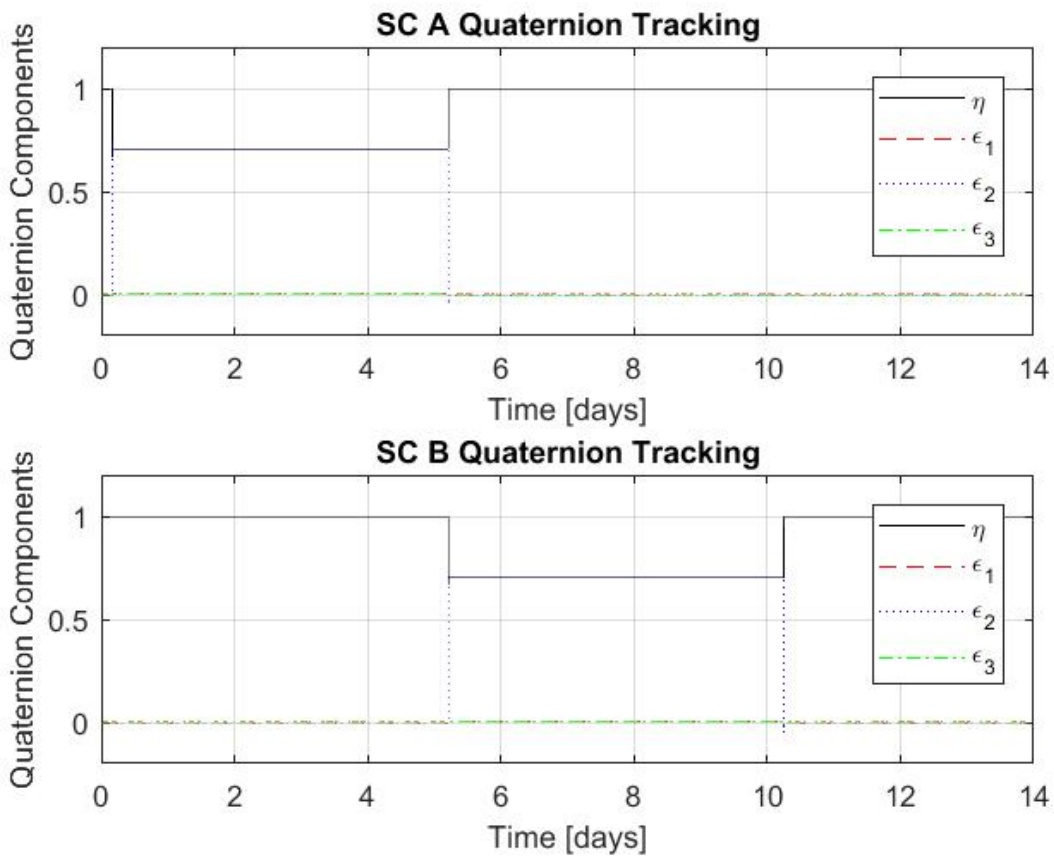

Figure A.6: Spacecraft quaternions over mission lifetime.
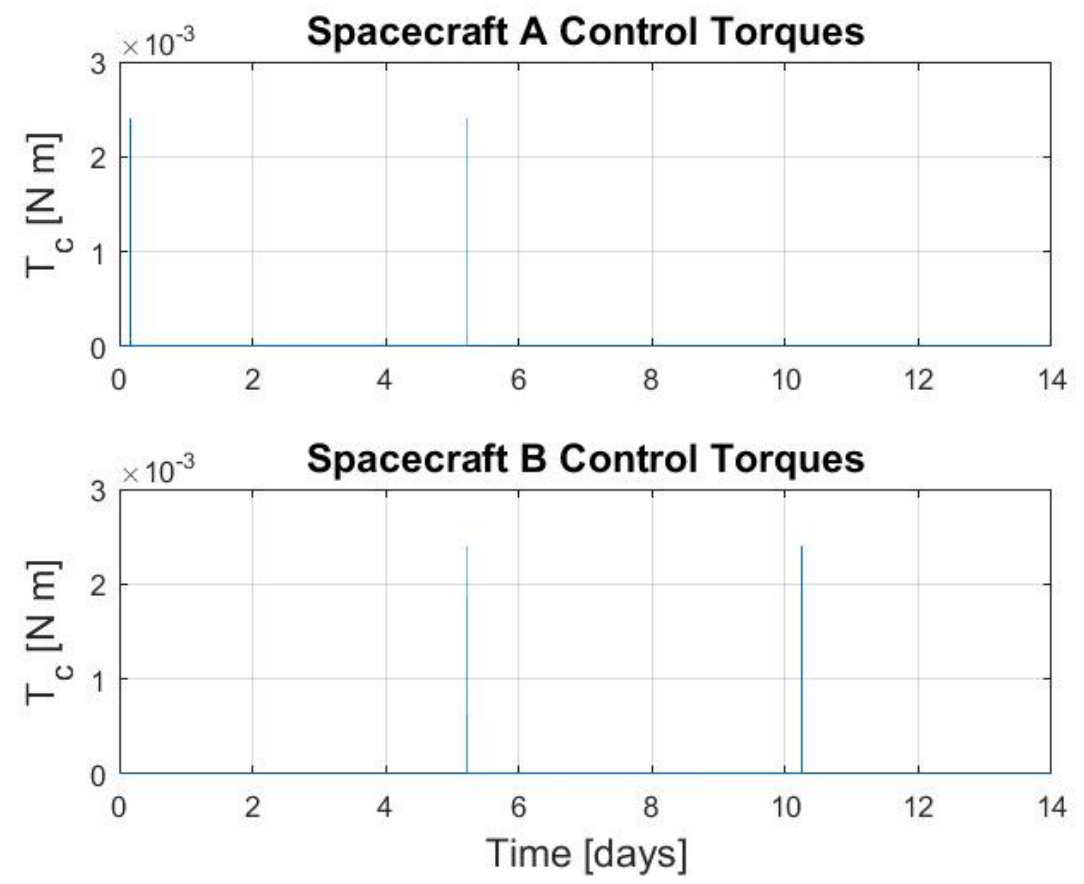

Figure A.7: Control torques required over mission lifetime. 
Appendix B

COMPLETE RESULTS FROM ALTERNATIVE SIMULATION

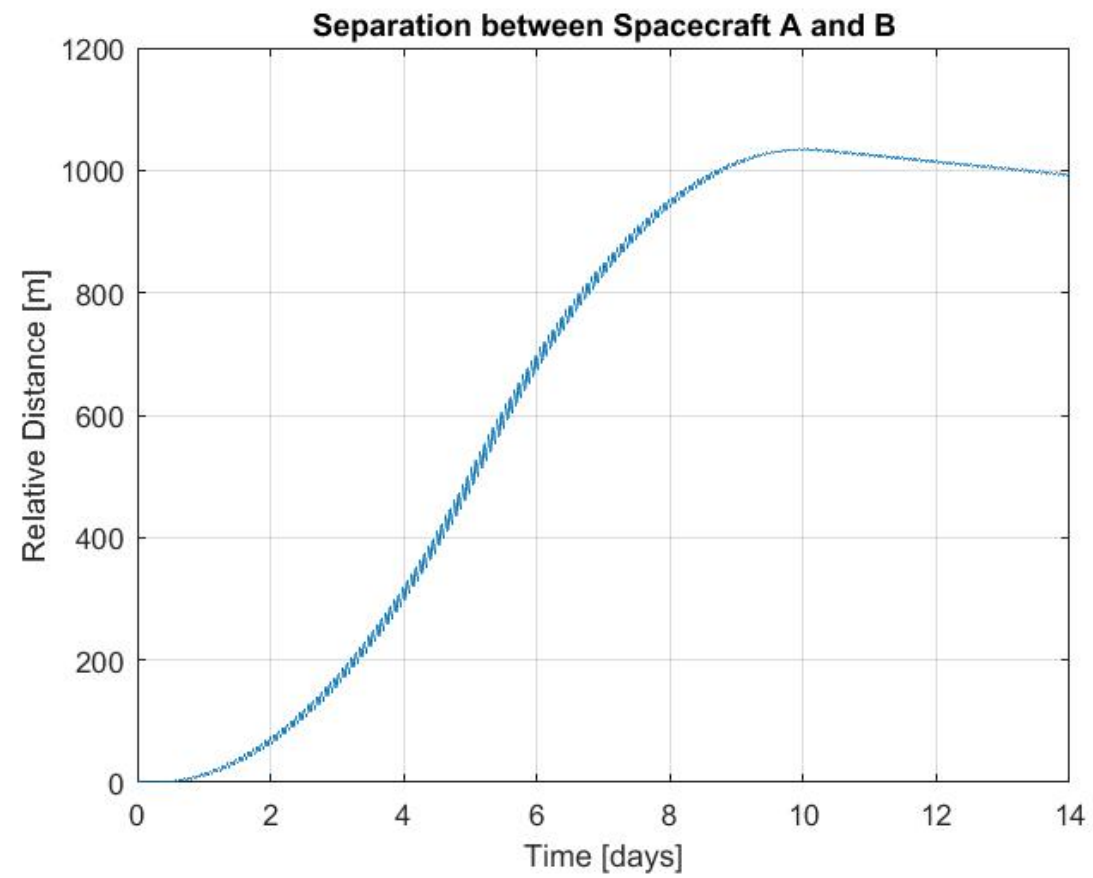

Figure B.1: Relative position of spacecraft over mission lifetime. 


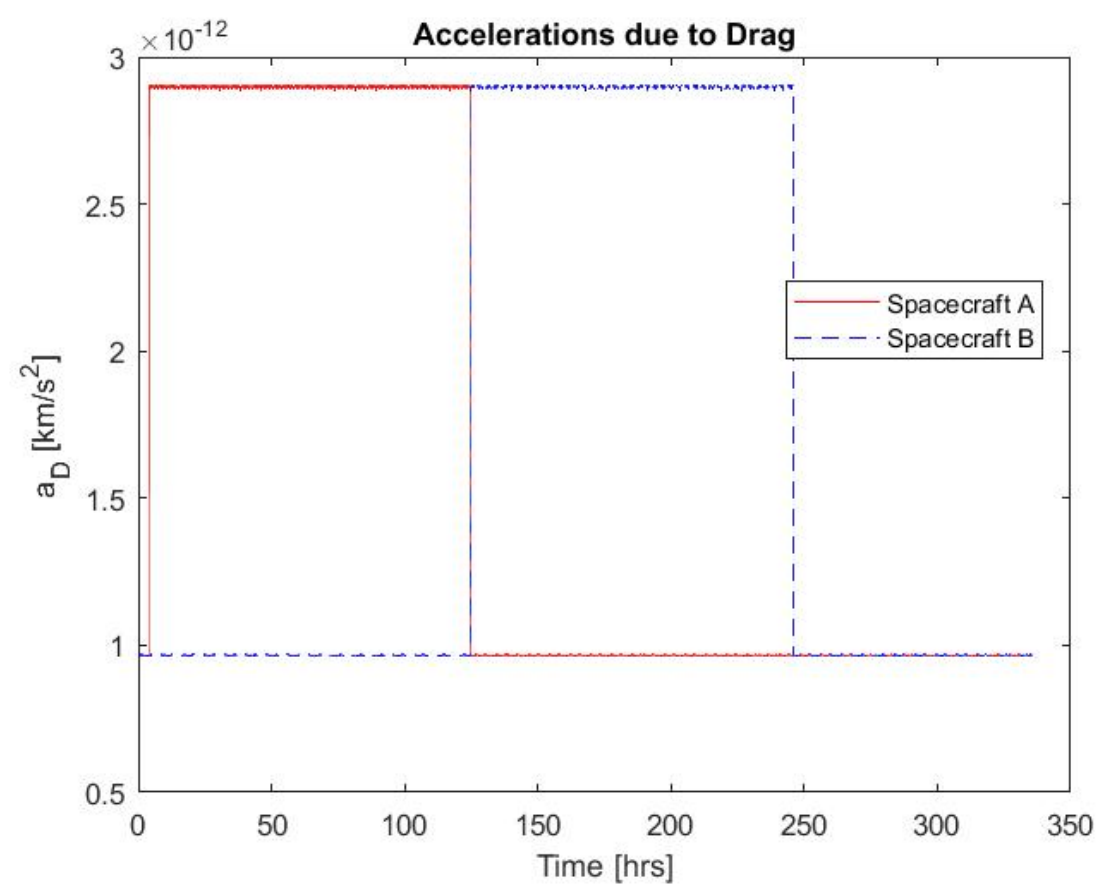

Figure B.2: Accelerations due to drag over mission lifetime.
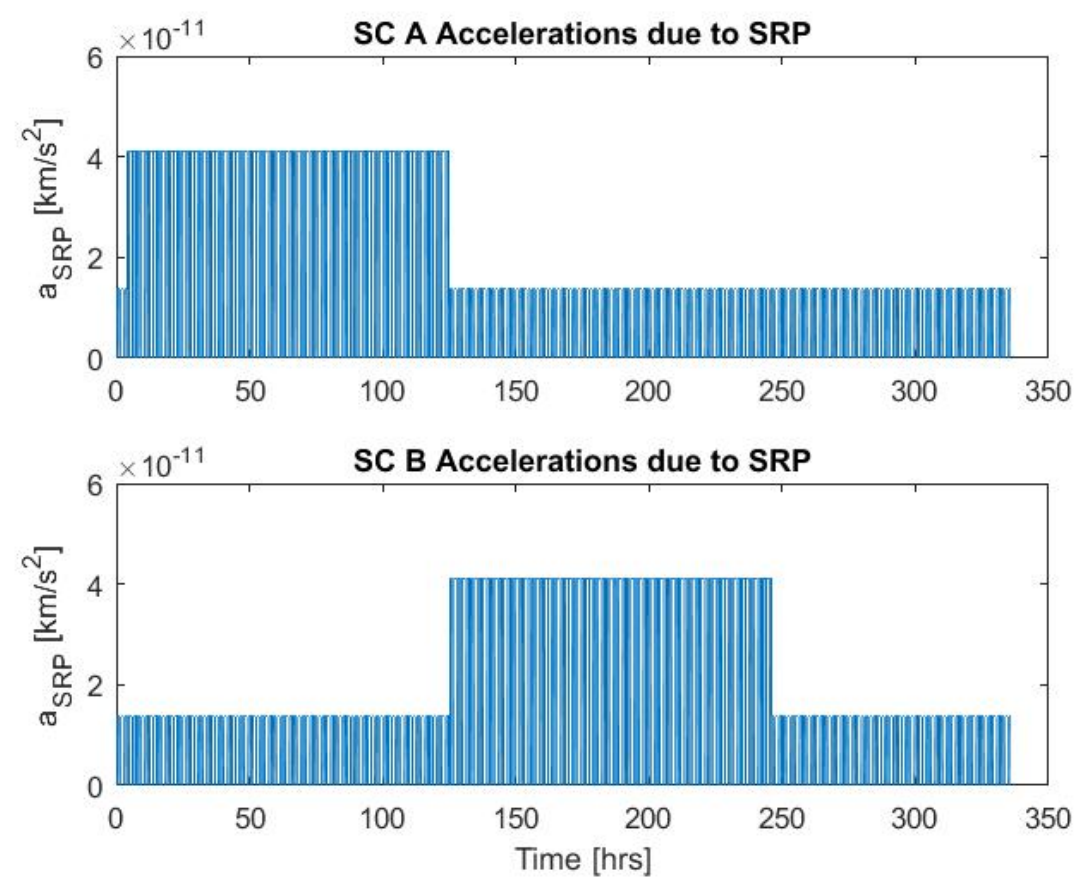

Figure B.3: Accelerations due to SRP over mission lifetime. 


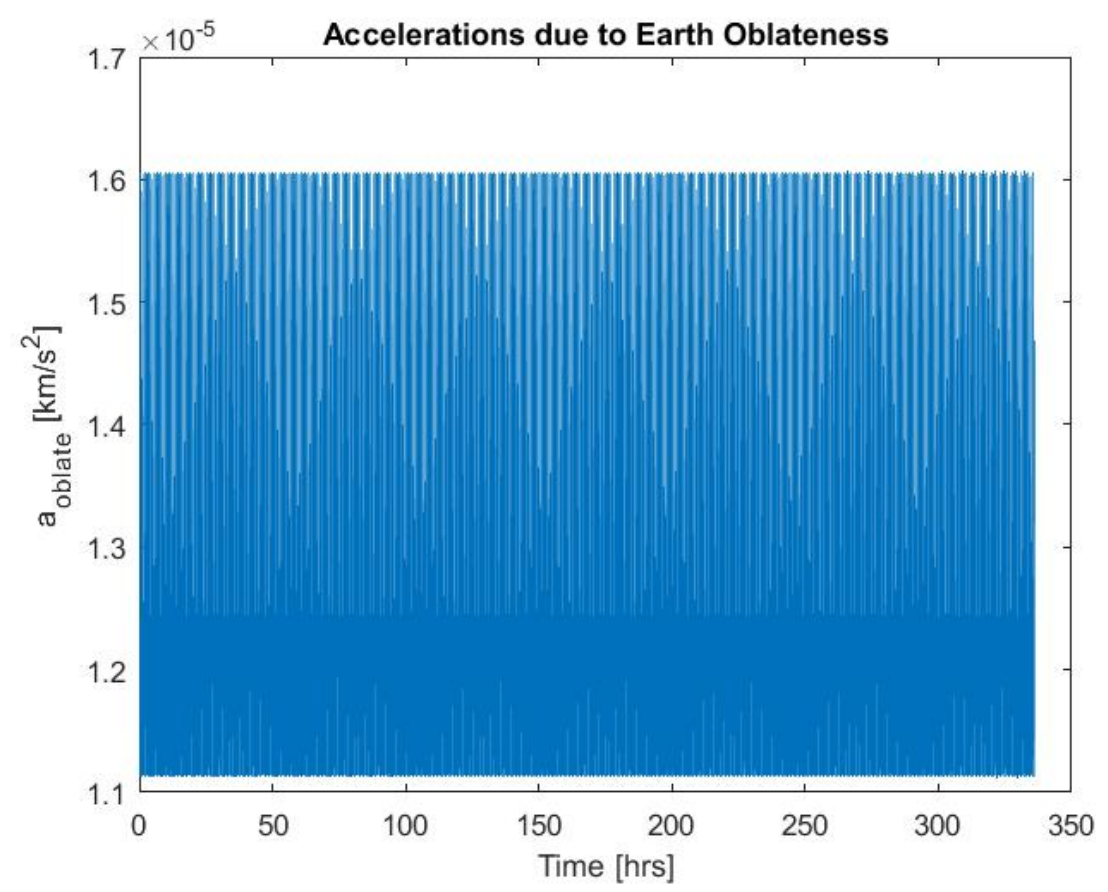

Figure B.4: Accelerations due to Earth oblateness over mission lifetime.

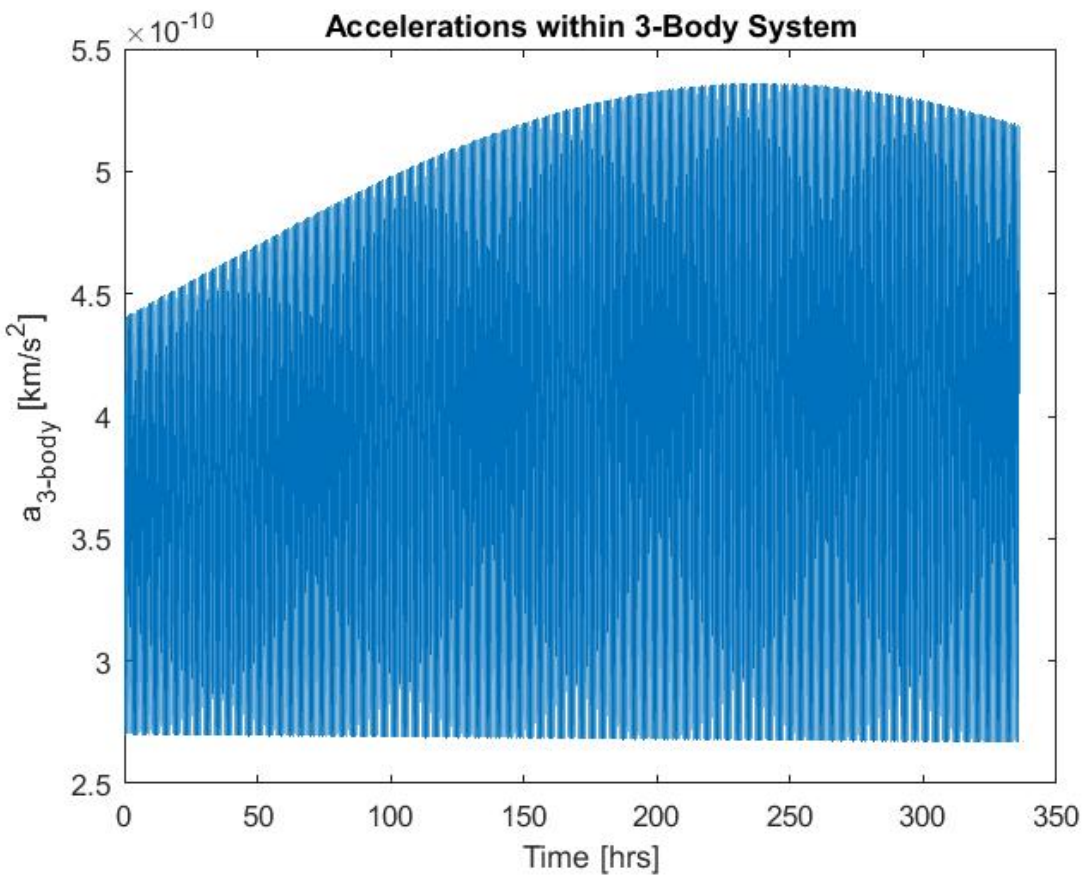

Figure B.5: Accelerations due to 3-body system over mission lifetime. 

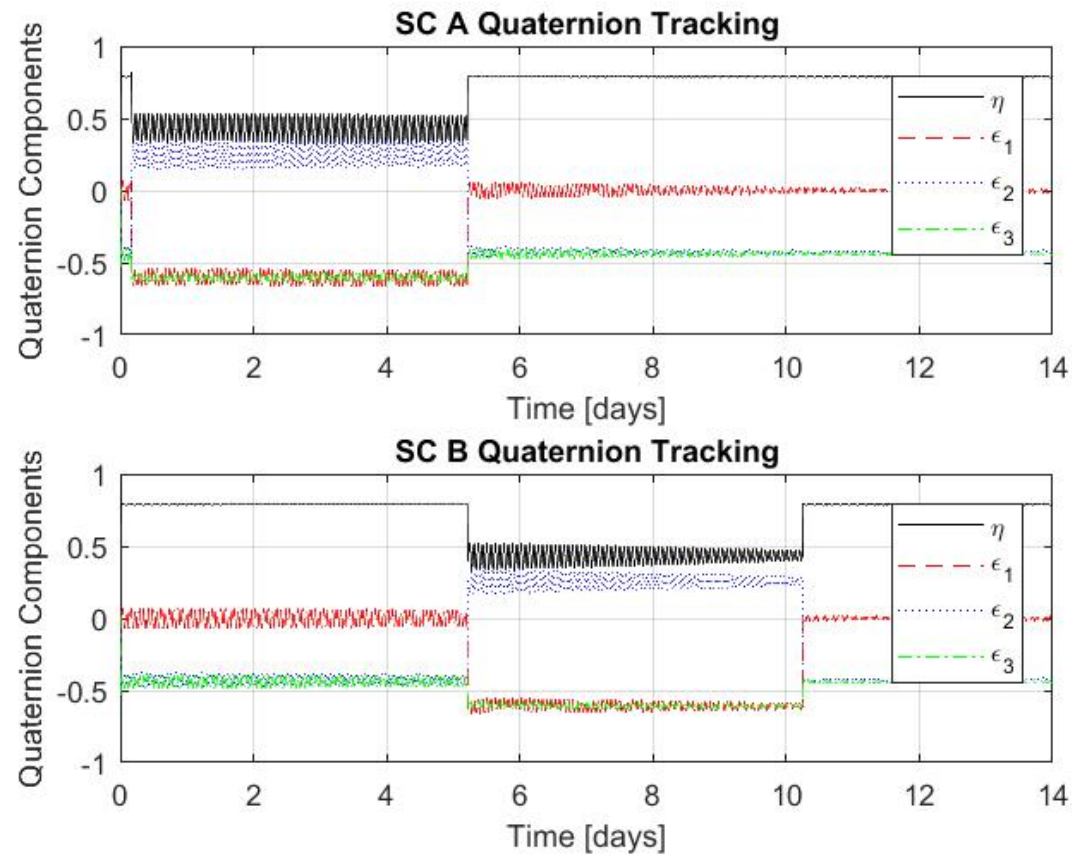

Figure B.6: Spacecraft quaternions over mission lifetime.
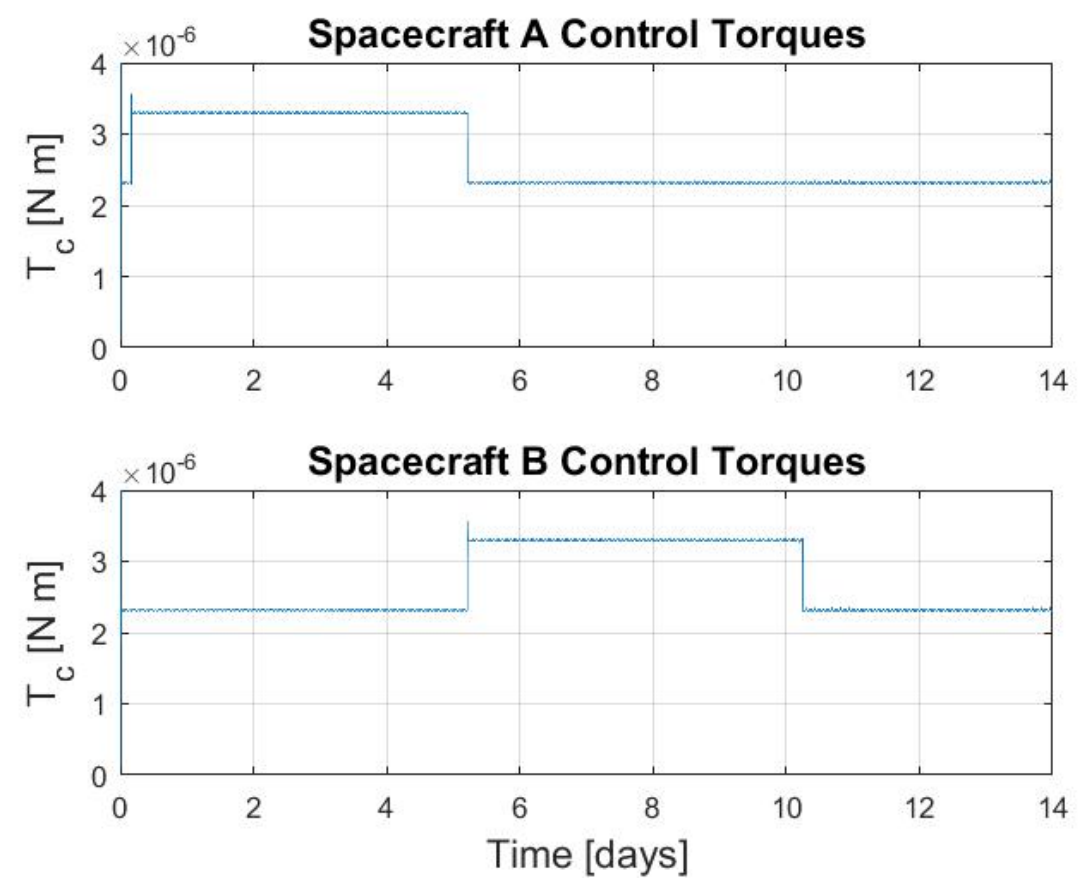

Figure B.7: Control torques required over mission lifetime. 